\title{
Risk-Dependent Centrality in Economic and Financial Networks*
}

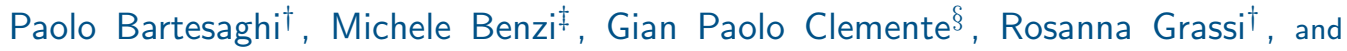 \\ Ernesto Estrada
}

\begin{abstract}
Node centrality is one of the most important and widely used concepts in the study of complex networks. Here, we extend the paradigm of node centrality in financial and economic networks to consider the changes of node "importance" produced not only by the variation of the topology of the system but also as a consequence of the external levels of risk to which the network as a whole is subjected. Starting from the "Susceptible-Infected" (SI) model of epidemics and its relation to the communicability functions of networks, we develop a series of risk-dependent centralities for nodes in (financial and economic) networks. We analyze here some of the most important mathematical properties of these risk-dependent centrality measures. In particular, we study the newly observed phenomenon of ranking interlacement, by means of which two entities may interlace their ranking positions in terms of risk in the network as a consequence of the change in the external conditions only, i.e., without any change in the topology. We test the risk-dependent centralities by studying two realworld systems: the network generated by collecting assets of the S\&P 100 and the corporate board network of the U.S. top companies, according to Forbes in 1999. We found that a high position in the ranking of the analyzed financial companies according to their risk-dependent centrality corresponds to companies more sensitive to the external market variations during the periods of crisis.
\end{abstract}

Key words. communicability, complex networks, SI models, centrality measures, risk propagation

AMS subject classifications. 05C82, 90B15, 91G80

DOI. $10.1137 / 19 \mathrm{M} 1302041$

1. Introduction. Modern economic and financial systems are characterized by a vast collection of interacting agents $[1,6,12,37,47,55]$. In economic systems, for instance, the interdependence among entities characterizes the trade and exchange of goods in nonanonymous markets as well as in risk-sharing agreements in developing countries [1]. In this framework, the agents' interaction is responsible for the nature of the relations between the individual behavior and the aggregate behavior [55].

The human factor which underlies these economic and financial systems is also characterized by the interconnectivity. The existence of networks of interpersonal relations has been empirically observed to constitute a fundamental factor in shaping the interinstitution networks, or in accounting for the networks of risk-sharing agreements [33, 34], the formation

\footnotetext{
${ }^{*}$ Received by the editors November 25, 2019; accepted for publication (in revised form) April 13, 2020; published electronically June 2, 2020.

https://doi.org/10.1137/19M1302041

${ }^{\dagger}$ Department of Statistics and Quantitative Methods, University of Milano - Bicocca, Via Bicocca degli Arcimboldi 8, 20126, Milano, Italy (paolo.bartesaghi@unimib.it, rosanna.grassi@unimib.it).

¥Scuola Normale Superiore, Piazza dei Cavalieri 7, 56126, Pisa, Italy (michele.benzi@sns.it).

$\S$ Department of Mathematics for Economics, Financial and Actuarial Sciences, Università Cattolica del Sacro Cuore, Largo Gemelli 1, 20123, Milano, Italy (gianpaolo.clemente@unicatt.it).

IInstitute of Mathematics and Applications (IUMA), Universidad de Zaragoza, Pedro Cerbuna 12, E-50009 Zaragoza, Spain; ARAID Foundation, Government of Aragón, 50018 Zaragoza, Spain (estrada66@unizar.es).
} 
of buyer-seller networks $[18,40,60]$, product adoption decisions [23, 54], diffusive processes [41, 46, 64], industrial organization [47], trade agreements [37], and even the existence of interbank networks [1]. This is not surprising as humans are responsible for the execution of deals between the institutions to which they belong [16, 59, 71].

From a mathematical perspective all these interdependencies between economic and financial entities can be captured by the formal concept of a network, in which nodes represent the entities (individuals, firms, countries, etc.) and edges account for the relations between such entities, ranging from social relations to trade agreements [29]. Hence, it is possible to use the tools of network theory to analyze the structure and evolution of these systems as well as the dynamic processes that take place among them. On one side, researchers have studied the topological properties of these networks (sometimes called static analysis), which do not assume mechanisms of transmission of effects through the economic and financial entities [38, 52, 79]. Among such studies, it is frequent to find analyses of clusters formed by groups of institutions, as well as the centrality of individual nodes in the networks [8, 13, 78]. Specifically, centrality measures (see Chapter 5 in [29] for a detailed analysis) are topological characterizations of the nodes and their neighborhood in a network. In the analysis of financial and economic networks, the use of centrality measures is not so effective, as the classical ones provide a static view of the network, and even other measures based on dynamic processes, such as random walk-based centralities [69], do not capture the changing conditions to which these networks could be subject in relatively short periods of time. As an illustrative example, let us consider a hypothetical interbank network for which we are interested in analyzing the risk-dependent exposure of the various entities of the system. Any centrality measure will point out a specific and static ranking of the nodes. However, a bank which is very central at a low level of external risk is not necessarily central when such a level of external risk increases, and vice versa. On the other hand, the propagation of shocks through these networks is considered, and this is usually known as dynamic analysis [3, 15, 17, 25, 39, 48, 43]. In these studies, a specific way of transmission of these shocks through the network is assumed - as in the case of "Susceptible-Infected" and "Susceptible-Infected-Recovered" epidemiological models $[63,67,73]$ - and then a systemic risk analysis is based on the contagion effects observed through such models.

In this work we develop a mathematical model to account for the risk exposure of an entity in a networked (economic or financial) system. This model is based on the relation between the Susceptible-Infected epidemiological model and the so-called communicability functions of a network [30]. Using this connection we derive new centrality indices that quantify the level of risk at which an entity is exposed to as a function of the global external level of risk. Our approach takes advantage of the benefits of both static and dynamic analyses. Indeed, unlike the standard approaches followed in the literature, these risk-dependent centralities are not static indices, as most centrality indices are, but they vary with the change of the external global risk level the system is subject to. More importantly, the ranking of the nodes in these networks also depends on this global external level of risk. This means that an entity - a node in the network - which is at a low (high) level of risk under external conditions can be at a high (low) level under different conditions.

We test our model by using two different systems, a network of assets based on the daily returns of the components of the S\&P 100 for the period ranging from January 2001 to 
December 2017 and a network representing the interconnection between companies in the U.S. top corporates according to Forbes in 1999. In the first case we extract the essential information about asset correlations through the minimum spanning tree. We measure how the centrality of the assets changes at different values of the external risk. What emerges is a high volatility in the rankings during the financial crisis of 2007-2008, when the node centrality proves to be more sensitive to the external risk. In the case of the corporate network we analyze a sample of significant companies, looking for a correlation between shareholder value creation (SVC) and behavior during and after the crisis period to which data refer. We find that a remarkable increase in their risk-centrality ranking during a crisis corresponds to a less resilient reaction to the external market turmoil.

The paper is structured as follows. In subsection 1.1 we recall the main literature about the use of epidemiological models for modeling financial contagion, and we motivate the choice of a Susceptible-Infected model. The necessary mathematical preliminaries are given in section 2. Therefore, we describe a Susceptible-Infected (SI) model on a financial network (section 3), and we define the risk-dependent centrality proving some mathematical properties (section 4). We perform numerical analyses of the proposed centrality for random networks (section 5), we apply the proposed measure to real-world financial networks (section 6), and we analyze the ranking interlacement problem (section 7). Section 8 comments on how the proposed model could provide additional insights into the analysis of the economic and financial impacts of the crisis related to the diffusion of the new coronavirus called SARS-CoV-2. Conclusions follow in section 9 .

1.1. Related literature and motivations. The process in which one financial institution spreads negative effects to another institution very much resembles the propagation of epidemics on networks $[66,48]$. The fact that such processes are known as "financial contagion" already captures part of these similarities. Then, it is not strange that epidemiological models are frequently used to capture the subtleties of financial contagion processes. There are many such compartmental models in epidemiology, but the most widely used for modeling financial contagion are the Susceptible-Infected-Recovered (SIR) [21, 36, 74, 58, 76, 42] and SusceptibleInfected-Susceptible (SIS) $[5,14]$ models. They are used not only to model financial contagion per se but also for the propagation of rumors and innovations of interest for financial institutions $[70,50]$. These models are well suited to depicting financial contagion because they do not require arbitrary assumptions on loss rates and balance sheets. As remarked by Toivanen [80], they capture the psychological aspects of the contagion process "by relating a bank's relative financial strength with the perceived counterparty risk and expectations."

The previously mentioned SIS/SIR models and their variants are mainly used in studying the dynamics of contagion in a system in a post-mortem way. As is well known, both SIS and SIR models are characterized by the presence of a threshold $\tau$, which is defined as the reciprocal of the principal eigenvalue $\lambda_{1}$ of the adjacency matrix. The below-the-threshold or above-the-threshold behavior of the spreading process depends on whether the effective infection rate is less than or greater than such a threshold. Below the threshold, we have the extinction of the contagion, and above the threshold a nonzero fraction of infected nodes persists in the network even over a wide range of timescales. The effective infection rate depends on both the infection rate per link $\gamma$ and on the curing or recovering rate $\delta$. For

Copyright (c) by SIAM. Unauthorized reproduction of this article is prohibited. 
instance, in Figure 1(a) we illustrate the evolution of a contagion dynamics for an ErdösRényi (ER) graph with 100 nodes and connection probability 0.1 by using the SIS model. The principal eigenvalue of the adjacency matrix is $\lambda_{1} \approx 10.71$ so that the epidemic threshold is $\tau \approx 0.093$. The infectivity rate per link is 0.002 for both curves, and the initial infection probability is 0.2 (20 nodes over 100 initially infected). The curing rate is 0.001 for the dashed red line (epidemic) and 0.04 for the solid blue line (extinction). Then, the effective infection rate is $2>0.093$ for the dashed red line (epidemic) and $0.05<0.093$ for the solid blue line (extinction).

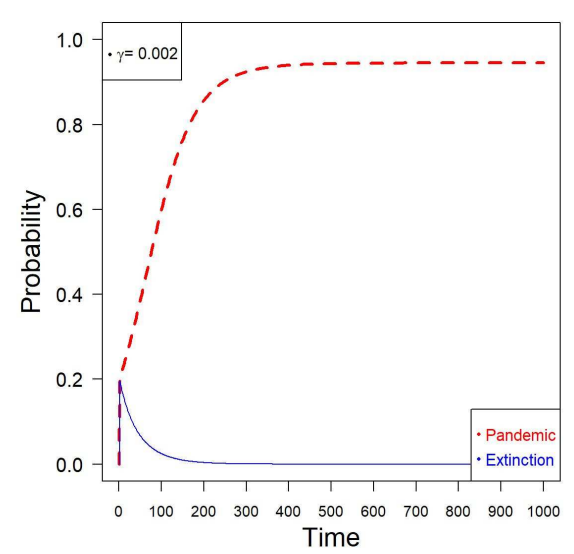

(a)

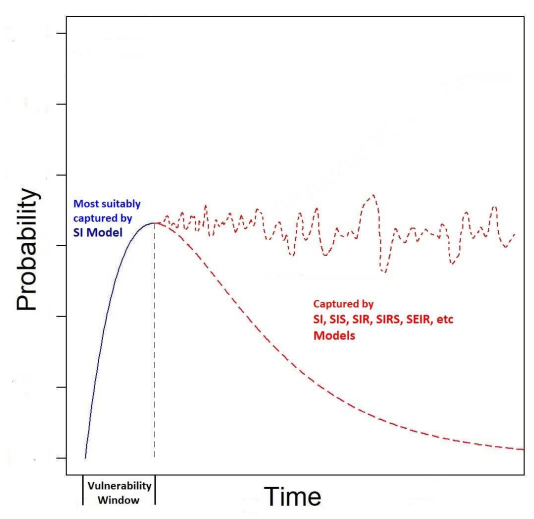

(b)

Figure 1. (a) Evolution of an SIS dynamics over an Erdös-Rényi (ER) graph. (b) Evolution of contagion before and after the window of vulnerability. Adapted from Lee, Tenneti, and Eun [62].

In this work we are interested in the very early signals that the system can provide for alerting us about a propagation of a financial contagion. In this case it is very important to consider the window of vulnerability between the time the contagion phenomenon is first recognized and the time an action is taken to face the infection. This window could be arbitrarily wide. In any real condition, there is a nonnegligible time interval in which a recovery tool is not available yet and the recovering rate is equal to zero. It has been recently shown by Lee, Tenneti, and Eun [62] that, within this window, the spreading phenomenon is better described by an SI model than by any other model with a nonnull recovering rate, e.g., SIR or SIS (see Figure 1(b)). In this framework, a key point is to predict the "most at risk" nodes in the network. Therefore, we are interested in the early times of the epidemic when it is possible to limit or avoid the distress propagation by introducing specific measures on the risky nodes in the network.

Moreover, in order to be effective in reducing the spreading phenomenon, the curing rate has to be large enough. More precisely, since $\lambda_{1}>\max \left(\bar{k}, \sqrt{k_{\max }}\right)(\bar{k}$ being the mean degree and $k_{\max }$ the maximum degree), the curing rate has to be at least $\delta>\gamma \cdot \max \left(\bar{k}, \sqrt{k_{\max }}\right)$ to get 
a below-the-threshold behavior. But for a big real network $\sqrt{k_{\max }}$ can be very large, even if the mean degree is small. This implies that $\delta$ has to be significantly bigger than $\gamma$ or, in other words, the infection significantly weaker than the self-recovering process. This fact could be totally unlikely in a real contagion process on a real network, and it makes the use of the SIS or SIR model extremely unrealistic, as remarked by Lee, Tenneti, and Eun [62]. Even when $\delta$ is small and the node infection process is dominant, the corresponding epidemic dynamic is better captured by the SI model. From an application point of view, this is possibly true over a wide range of timescales under constrained environments where applying massive action to limit contagion is practically infeasible.

As mentioned before, the detection of risky nodes in a network could be relevant for limiting the risk propagation effects (see, e.g., $[44,7]$ ). Hence, the centrality of a given institution as best spreader node in a contagion process has been widely explored (see, for instance, [63]) in order to identify the most dangerous crisis epicenter. The idea of best spreader node has also been studied in [81] in terms of topological centralities, which was previously investigated under the name "vibrational centrality" (see, e.g., [31]). Centralities have been also used as measures to assess contagion in the interbank market. In this framework, Dimitrios and Vasileios [22] recommended the use of well-established centrality measures as a way to identify the most important variables in a network. Battiston et al. [8] introduced DebtRank, a centrality measure that accounts for distress in one or more banks, based on the possibility of losses occurring prior to default. The concept that some banks might be too central to fail originates from this work (see, e.g., [8]).

2. Preliminaries. Here we use the terms "graphs" and "networks" interchangeably. Most of the network theoretic concepts defined hereafter can be found in [29]. A graph $\Gamma=(V, E)$ is defined by a set of $n$ nodes (vertices) $V$ and a set of $m$ edges $E=\{(u, v) \mid u, v \in V\}$ between the nodes. $(u, u) \in E$ is a loop starting and ending in $u$. The degree of a node, denoted by $k_{u}$, is the number of edges incident to $u$ in $\Gamma$. The adjacency matrix of the graph $A=\left(A_{u v}\right)_{n \times n}$ with entries $A_{u v}=1$ if $(u, v) \in E$ or zero otherwise. We consider here simple graphs, i.e., without loops and multiedges. The theoretical model will be developed for unweighted networks; we also recall here the definition of weighted graphs, as we consider in the paper two empirical real examples for which the network is weighted. A weighted graph $\Gamma^{\prime}=(V, E, W)$ is a graph in which $w_{u v} \in W$ is a positive number assigned to the corresponding edge $(u, v) \in E$. In this case the sum of the weights for all edges incident to a node is known as the weighted degree or strength. We consider here only undirected networks, such that $(u, v) \in E$ implies that $(v, u) \in E$. In this case the matrix $A$ can be expressed as $A=U \Lambda U^{T}$, where $U=\left[\vec{\psi}_{1} \cdots \vec{\psi}_{n}\right]$ is an orthogonal matrix of the eigenvectors of $A$ and $\Lambda$ is the diagonal matrix of eigenvalues $\lambda_{1} \geq \lambda_{2} \geq \cdots \geq \lambda_{n}$. The entries of $\vec{\psi}_{j}$ are denoted by $\psi_{j, 1}, \ldots, \psi_{j, n}$.

An important quantity for studying communication processes in networks is the communicability function [30], defined for a pair of nodes $u$ and $v$ as

$$
G_{u v}=\sum_{k=0}^{\infty} \frac{\left(A^{k}\right)_{u v}}{k !}=(\exp (A))_{u v}=\sum_{j=1}^{n} e^{\lambda_{j}} \psi_{j, u} \psi_{j, v} .
$$

It counts the total number of walks starting at node $u$ and ending at node $v$, weighted in decreasing order of their length by a factor of $\frac{1}{k !}$. A walk of length $k$ in $\Gamma$ is a set of 
nodes $i_{1}, i_{2}, \ldots, i_{k}, i_{k+1}$ such that for all $1 \leq l \leq k,\left(i_{l}, i_{l+1}\right) \in E$. A closed walk is a walk for which $i_{1}=i_{k+1}$. Therefore, $G_{u v}$ is considering shorter walks as more influential than longer ones. The matrix exponential is an example of a general class of matrix functions which are expressible as

$$
(f(A))_{u v}=\sum_{k=0}^{\infty} c_{k}\left(A^{k}\right)_{u v},
$$

where $c_{k}$ are coefficients giving more weight to the shorter than to the longer walks, and making the series convergent. The term $G_{u u}$, which counts the number of closed walks starting at the node $u$ giving more weight to the shorter than to the longer ones, is known as the subgraph centrality of the node $u$.

We also consider here a Susceptible-Infected (SI) model over an undirected network. Each susceptible node becomes infected at the infection rate $\gamma$ per link times the number of infected neighboring nodes. Let $t^{*}$ be the instant in which a node $i$ is infected. Node $i$ remains in this state $\forall t \geq t^{*}$ and does not come back susceptible. Let us introduce a random variable $X_{i}(t)$ denoting the state of a node $i$ at time $t$ :

$$
X_{i}(t)=\left\{\begin{array}{cc}
1 & \text { if } t \geq t^{*} \\
0 & \text { otherwise }
\end{array}\right.
$$

Then we define

$$
x_{i}(t)=P\left[X_{i}(t)=1\right]=\mathbb{E}\left[X_{i}(t)\right] \in[0,1],
$$

which is the probability that node $i$ is infected at time $t$. In other words, node $i$ is healthy at time $t$ with probability $1-x_{i}(t)$. For the whole network, we define the vector of probabilities:

$$
\vec{x}(t)=\left[x_{1}(t), \ldots, x_{n}(t)\right]^{T} .
$$

3. Model. Let us consider an SI model on a financial network. The nodes of a graph $\Gamma=$ $(V, E)$ represent financial institutions, and the edges connecting them represent an interaction that can transmit a "disease" from one institution to another. A node can be susceptible and then get infected from a nearest neighbor, or it is infected and can transmit the infection to other susceptible nodes. Let $\gamma$ be the infection rate, and let $x_{i}(t)$ be the probability that node $i$ gets infected at time $t$ from any infected nearest neighbor. Then,

$$
\frac{d x_{i}(t)}{d t}=\overrightarrow{\dot{x}}(t)=\gamma\left[1-x_{i}(t)\right] \sum_{j=1}^{n} A_{i j} x_{j}(t),
$$

which in matrix-vector form becomes

$$
\overrightarrow{\dot{x}}(t)=\gamma[1-\operatorname{diag}(\vec{x}(t))] A \vec{x}(t),
$$

with initial condition $\vec{x}(0)=\vec{x}_{0}$.

It is well known that on a strongly connected network ${ }^{1}[67]$

\footnotetext{
${ }^{1} \mathrm{~A}$ graph $\Gamma=(V, E)$ is strongly connected if and only if for each pair of nodes $i, j \in V$ there is a directed walk starting at $i$ and ending at $j$, and a directed walk starting at $j$ and ending at $i$.
}

Copyright (C) by SIAM. Unauthorized reproduction of this article is prohibited. 
1. if $\vec{x}_{0} \in[0,1]^{n}$, then $\vec{x}(t) \in[0,1]^{n}$ for all $t>0$;

2. $\vec{x}(t)$ is monotonically nondecreasing in $t$;

3 . there are two equilibrium points: $\vec{x}=\overrightarrow{0}$, i.e., no epidemic, and $\vec{x}=\overrightarrow{1}$ (the vector of all ones), i.e., full contagion;

4. the linearization of the model around the point $\overrightarrow{0}$ is given by

$$
\overrightarrow{\dot{x}}(t)=\gamma A \vec{x}(t),
$$

and it is exponentially unstable; in fact, since, in a nonempty undirected graph, $A$ has at least one positive eigenvalue, any solution component in the direction of the corresponding eigenvector grows unboundedly as $t$ increases;

5. each trajectory with $\vec{x}_{0} \neq \overrightarrow{0}$ converges asymptotically to $\vec{x}=\overrightarrow{1}$; i.e., the epidemic spreads monotonically to the entire network.

In particular, the linearized problem comes from the following observation. It can be checked that

$$
\dot{x}_{i}(t)=\gamma\left[1-x_{i}(t)\right] \sum_{j=1}^{n} A_{i j} x_{j}(t) \leq \gamma \sum_{j=1}^{n} A_{i j} x_{j}(t)
$$

or

$$
\overrightarrow{\dot{x}}(t) \leq \gamma A \vec{x}(t)
$$

$\forall i$ and $\forall t$. Then, we can use the linear dynamical system

$$
\overrightarrow{\dot{x}}^{\star}(t)=\gamma A \vec{x}^{\star}(t)
$$

as an upper bound for the original nonlinear dynamical system that has been used in the literature (see [67]) as an approximation of the exact problem. One of its main advantages is that it can be solved analytically and its solution $\vec{x}^{\star}(t)$ can be written as

$$
\vec{x}^{\star}(t)=e^{\gamma t A} \vec{x}_{0}^{\star},
$$

which using the spectral decomposition of $A$ can be written as

$$
\vec{x}^{\star}(t)=\sum_{j=1}^{n} e^{\gamma t \lambda_{j}} \vec{\psi}_{j} \vec{\psi}_{j}^{T} \vec{x}_{0}^{\star} .
$$

This solution to the linearized model is affected by the following main problems:

1. $\vec{x}^{\star}(t)$ grows quickly without bound in spite of the fact that $\vec{x}^{\star}(t)$ is a vector of probabilities which should not exceed the unit;

2. $\vec{x}^{\star}(t)$ is an accurate solution to the nonlinear SI problem only if $t \rightarrow 0$ and $\vec{x}_{0}^{\star} \rightarrow 0$.

The mathematical properties of the linear dynamical system (3.3) as well as of the solution (3.7) have been extensively studied by Mugnolo in [68]. We direct the reader to this reference for the details. 
Hereafter we will follow the recent work of Lee, Tenneti, and Eun [62], who proposed the following change of variable to avoid the aforementioned problems with the solution of the linearized SI model:

$$
y_{i}(t):=-\log \left(1-x_{i}(t)\right),
$$

which is an increasing convex function. Then, as $1-x_{i}(t)$ is the probability that node $i$ is not infected at a given time $t$, the new variable $y_{i}(t)$ can be interpreted as the information content of the node $i$ or the surprise of not being infected (see, e.g., [19]). According to [62], the SI model (3.1) can be now written as

$$
\frac{d y_{i}(t)}{d t}=\dot{y}_{i}(t)=\gamma \sum_{j=1}^{n} A_{i j} x_{i}(t)
$$

or

$$
\overrightarrow{\dot{y}}(t)=\gamma A \vec{x}(t) .
$$

The approximate solution to the SI model provided by [62] is then given by

$$
\vec{x}(t)=\overrightarrow{1}-e^{-\vec{y}(t)},
$$

where $e^{-\vec{y}(t)}$ is the vector in which the $i$ th entry is $e^{-y_{i}(t)}$ and

$$
\begin{aligned}
\vec{y}(t)= & e^{\gamma t A \operatorname{diag}\left(\overrightarrow{1}-\vec{x}_{0}\right)}\left[-\log \left(1-\vec{x}_{0}\right)\right] \\
& +\sum_{j=0}^{\infty} \frac{(\gamma t)^{j+1}}{(j+1) !}\left[A \operatorname{diag}\left(\overrightarrow{1}-\vec{x}_{0}\right)\right]^{j} A\left(\vec{x}_{0}+\left(\overrightarrow{1}-\vec{x}_{0}\right) \log \left(\overrightarrow{1}-\vec{x}_{0}\right)\right) .
\end{aligned}
$$

As stressed by [62], the interesting case of the dynamics is when $\vec{x}_{0}<\overrightarrow{1}$, in which case the solution simplifies to

$$
\vec{y}(t)=\vec{y}_{0}+\left[e^{\gamma t A \operatorname{diag}\left(\overrightarrow{1}-\vec{x}_{0}\right)}-I\right] \cdot \operatorname{diag}\left(\overrightarrow{1}-\vec{x}_{0}\right)^{-1} \vec{x}_{0} .
$$

Now, we can make the further assumption that the initial probabilities of being infected are equal for every node, i.e., that at the beginning every node has the same probability $\beta$ to be infected and to be the one from which the epidemic starts. This means that we are asking for

$$
x_{0 i}=\beta=\frac{c}{n} \quad \forall i=1, \ldots, n,
$$

for some scalar constant $c$. In this case $\operatorname{diag}\left(\overrightarrow{1}-\vec{x}_{0}\right)=\left(1-\frac{c}{n}\right) I=(1-\beta) I$. If we set $\alpha=1-\beta$, the approximate solution of the SI on the network becomes

$$
\vec{y}(t)=\vec{y}_{0}+\frac{1-\alpha}{\alpha}\left[e^{\alpha \gamma t A}-I\right] \overrightarrow{1},
$$


and since $\vec{y}_{0}=(-\log \alpha) \overrightarrow{1}$,

$$
\vec{y}(t)=\left(\frac{1}{\alpha}-1\right) e^{\alpha \gamma t A} \overrightarrow{1}-\left(\log \alpha+\frac{1-\alpha}{\alpha}\right) \overrightarrow{1} .
$$

The component $\left(e^{\alpha \gamma t A} \overrightarrow{1}\right)_{i}$ is called the total communicability of node $i$ and will be denoted by $\mathscr{R}_{i}$. Hence, componentwise we have

$$
y_{i}(t)=\left(\frac{1}{\alpha}-1\right) \mathscr{R}_{i}-\left(\log \alpha+\frac{1-\alpha}{\alpha}\right) .
$$

Keeping in mind that $-\log \alpha=y_{i}(0)$ and $\alpha=1-\beta$, we can also write the previous equation as

$$
\Delta y_{i}(t)=y_{i}(t)-y_{i}(0)=\frac{\beta}{\alpha}\left(\mathscr{R}_{i}-1\right),
$$

which means that $\mathscr{R}_{i}-1$ at time $t$ is proportional to the variation in the information content of node $i$ from time 0 to time $t$. Finally, the probability of node $i$ being infected at time $t$ can be expressed in terms of $\mathscr{R}_{i}$ as

$$
x_{i}(t)=1-(1-\beta) e^{-\frac{\beta}{1-\beta}\left(\mathscr{R}_{i}-1\right)} .
$$

When the parameter $\beta$ is fixed, the number of infected nodes depends only on the term $e^{\alpha \gamma t A} \overrightarrow{1}$ and then on the total communicabilities $\mathscr{R}_{i}$. It is worth noticing that the probability given by (3.20) for a node $i$ represents an upper bound for the exact solution of the SI model. Hence, in this way we do not underestimate the contagion probabilities. Let us consider, for instance, the time evolution of an infection propagation on an ER network with 100 nodes and edge density $\delta=0.1$. Results are illustrated in Figure 2 for two different values of the infectivity rate, $\gamma=0.001$ (left) and $\gamma=0.002$ (right). The dashed red lines represent the mean probability that a node is infected at time $t$ as given by (3.20). The solid blue lines represent the same probability as given by the exact solution of the Kermack-McKendrick SI model with the same mean degree. In both plots, the initial probability is $\beta=0.01$.

4. Risk-dependent centrality. Let us designate $\zeta=\alpha \gamma t$, which determines the level of risk to which the whole network is subject at time $t$. For instance, for $\gamma=0$, i.e., $\zeta=0$, there is no risk of infection on the network as a node cannot transmit the disease to a nearest neighbor. This situation corresponds to the case of isolated nodes (no edges). When $\zeta \rightarrow \infty$ the risk of infection is very high due to the fact that for a fixed value of $c$ the infectivity is infinite. Therefore, we call $\mathscr{R}_{i}=\left(e^{\zeta A} \overrightarrow{1}\right)_{i}$ the risk-dependent centrality of the node $i$. That is, the value of $\mathscr{R}_{i}$ reflects how central a node is in "developing" the epidemics on the network. As the networks considered are undirected, this centrality accounts for both the facility with which the node gets infected as well as the propensity of this node to infect other nodes. The index $\mathscr{R}_{i}$ can be expressed as

$$
\mathscr{R}_{i}=\left[\left(I+\zeta A+\zeta^{2} \frac{A^{2}}{2 !}+\zeta^{3} \frac{A^{3}}{3 !}+\cdots\right) \overrightarrow{1}\right]_{i},
$$

Copyright $\odot$ by SIAM. Unauthorized reproduction of this article is prohibited. 


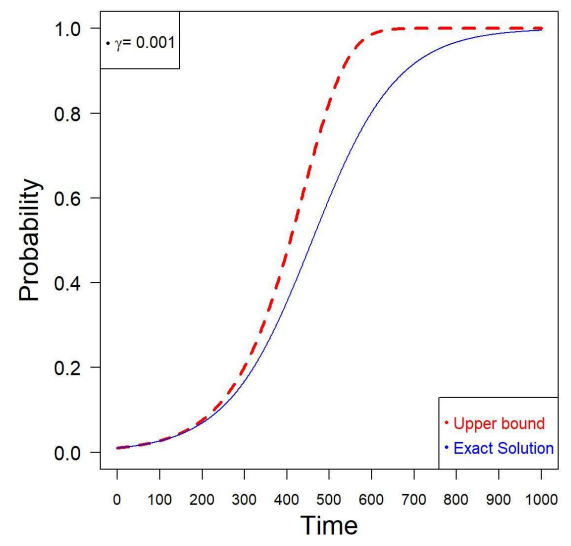

(a)

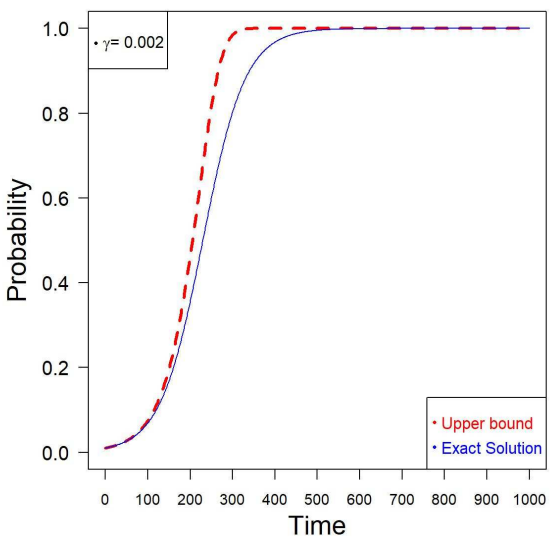

(b)

Figure 2. Simulation of the progression of an SI epidemic on an ER network with 100 nodes and edge density $\delta=0.1$. The parameters used in the model are $\beta=0.01$ and $\gamma=0.001$ (left) and $\gamma=0.002$ (right). Dashed (red) lines represent the upper bound given by (3.20); solid (blue) lines represent the value of the same probability in a Kermack-McKendrick SI model with the same mean degree $\bar{k}=(n-1) \delta$.

which indicates that it counts the number of walks of different lengths, which have started at the corresponding node, weighted by a factor $\frac{\zeta^{k}}{k !}$. It is straightforward to realize from the definition of the risk-dependent centrality that it can be split into two contributions. That is, $\mathscr{R}_{i}$ is composed of a weighted sum of all closed walks that start and end at $i,\left(e^{\zeta A}\right)_{i i}$, and of the weighted sum of walks that start at the node $i$ and end elsewhere, $\sum_{j \neq i}\left(e^{\zeta A}\right)_{i j}$,

$$
\mathscr{R}_{i}=\left(e^{\zeta A}\right)_{i i}+\sum_{j \neq i}\left(e^{\zeta A}\right)_{i j}:=\mathscr{C}_{i}+\mathscr{T}_{i}
$$

where the first term in the right-hand side represents the circulability of the disease around a given node and the second one represents the transmissibility of the disease from the given node to any other in the network. The circulability is very important because it accounts for the ways the disease has to become endemic. For instance, a large circulability for a node $i$ implies that the disease can infect its nearest neighbors and will keep coming back to $i$ over and over again in a circular way. We start now by proving some results about these riskdependent centralities. The following theorem is a special case of results found, for instance, in [11].

Theorem 4.1. The node ranking given by the risk-dependent centralities $\mathscr{R}_{i}(\zeta)$, with $i=$ $1, \ldots, n$, reduces to the ranking given by the degree $k_{i}$ in the limit as the risk $\zeta \rightarrow 0$, and to the ranking given by eigenvector centrality as $\zeta \rightarrow \infty$.

Proof. We begin by observing that the ranking of nodes, in terms of their risk-dependent centrality, is unaffected if all the centralities $\mathscr{R}_{i}$ are shifted and rescaled by the same amount. 
That is, the same ranking is obtained using either $\mathscr{R}_{i}$ or the equivalent measure

$$
\hat{\mathscr{R}}_{i}=\frac{\mathscr{R}_{i}-1}{\zeta}
$$

where $\zeta>0$. Now, we have

$$
\hat{\mathscr{R}}_{i}=\left[\left(A+\frac{\zeta}{2 !} A^{2}+\cdots\right) \overrightarrow{1}\right]_{i}=k_{i}+\frac{\zeta}{2 !}\left(A^{2} \overrightarrow{1}\right)_{i}+O\left(\zeta^{2}\right) .
$$

Hence, in the limit of $\zeta \rightarrow 0$, the ranking given by $\mathscr{R}_{i}$ is identical to degree ranking.

To study the limit for $\zeta$ large we write

$$
\mathscr{R}_{i}=\left[e^{\zeta A \overrightarrow{1}}\right]_{i}=\sum_{k=1}^{n} e^{\zeta \lambda_{k}}\left(\psi_{k}^{T} \overrightarrow{1}\right) \psi_{k, i}=e^{\zeta \lambda_{1}}\left(\psi_{1}^{T} \overrightarrow{1}\right) \psi_{1, i}+\sum_{k=2}^{n} e^{\zeta \lambda_{k}}\left(\psi_{k}^{T} \overrightarrow{1}\right) \psi_{k, i} .
$$

We note again that for ranking purposes we can use the equivalent measure obtained by dividing all risk-dependent centralities by the same quantity, $e^{\zeta \lambda_{1}}\left(\psi_{1}^{T} \overrightarrow{1}\right)$, which is strictly positive. That is, we can use

$$
\tilde{\mathscr{R}}_{i}=\psi_{1, i}+\frac{1}{\psi_{1}^{T} \overrightarrow{1}} \sum_{k=2}^{n} e^{\zeta\left(\lambda_{k}-\lambda_{1}\right)}\left(\psi_{k}^{T} \overrightarrow{1}\right) \psi_{k, i} .
$$

Since the network is connected, the Perron-Frobenius theorem ensures that $\lambda_{1}>\lambda_{2} \geq \cdots \geq$ $\lambda_{n}$. Hence, each term $e^{\zeta\left(\lambda_{k}-\lambda_{1}\right)}$ for $k=2, \ldots, n$ vanishes in the limit as $\zeta \rightarrow \infty$, and we see from (4.5) that the risk-dependent centrality measure gives the same ranking as eigenvector centrality for $\zeta$ large.

It is interesting to observe that the risk-dependent centrality of every node also depends on the (strictly positive) quantity

$$
\psi_{1}^{T} \overrightarrow{1}=\sum_{j=1}^{n} \psi_{1, j}
$$

see (4.4). The larger this quantity is, the higher the risk-dependent centrality of each node is. Assuming that the dominant eigenvector is normalized so as to have Euclidean norm equal to 1 , it is well known that this quantity is always between 1 and $\sqrt{n}$. The value 1 is never attained for a connected graph. It can only be approached in the limit as all of the eigenvector centrality is concentrated on one node, say node $i$, where it takes values arbitrarily close to 1 , with the values $\psi_{1, j}$ for all $j \neq i$ taking arbitrarily small values. An example of this would be the star graph ${ }^{2} S_{n}$ for $n \rightarrow \infty$. The maximum value is attained in the case where all nodes have the same eigenvector centrality: $\psi_{1,1}=\psi_{1,2}=\cdots=\psi_{1, n}$ (i.e., in the case of regular graphs).

Let us return to the decomposition $\mathscr{R}_{i}=\mathscr{C}_{i}+\mathscr{T}_{i}$ of the risk-dependent centrality of a node into its two components, circulability and transmissibility. Similar considerations apply to these quantities. We summarize them in the following result.

\footnotetext{
${ }^{2}$ We recall that the star graph $S_{n}$ consists of $n-1$ nodes $v_{1}, \ldots, v_{n-1}$, each attached to a central node $v_{n}$ by an edge.
}

Copyright (C) by SIAM. Unauthorized reproduction of this article is prohibited. 
Theorem 4.2. The node rankings given by the degree $k_{i}$ and the eigenvector centrality are obtained as limiting cases of the risk-dependent circulability $\mathscr{C}_{i}(\zeta)$ as the external level of risk $\zeta$ decreases to zero or increases to infinity, respectively. The same is true for the risk-dependent transmissibility $\mathscr{T}_{i}(\zeta)$.

Proof. The proof for the circulability is a straightforward adaptation of that for the total communicability; see also [11].

We give the details for the transmissibility, which has not been analyzed before. We have for $i \neq j$ that

$$
\left(e^{\zeta A}\right)_{i j}=\zeta A_{i j}+\frac{\zeta^{2}}{2 !} w_{i, j}^{(2)}+O\left(\zeta^{3}\right)
$$

where $w_{i, j}^{(2)}$ denotes the number of walks of length two between node $i$ and node $j$. Dividing by $\zeta>0$, summing over all $j \neq i$, and taking the limit as $\zeta \rightarrow 0$, we find

$$
\zeta^{-1} \mathscr{T}_{i}=\zeta^{-1} \sum_{j \neq i}\left(e^{\zeta A}\right)_{i j} \rightarrow \sum_{j \neq i} A_{i j}=k_{i}
$$

where we have used the fact that $A_{i i}=0$, for all $i$. Hence, transmissibility is equivalent to node degree in the small $\zeta$ limit. For the large $\zeta$ limit we write

$$
\mathscr{T}_{i}=\sum_{j \neq i} \sum_{k=1}^{n} e^{\zeta \lambda_{k}} \psi_{k, i} \psi_{k, j}=e^{\zeta \lambda_{1}} \psi_{1, i} \sum_{j \neq i} \psi_{1, j}+\sum_{k=2}^{n} e^{\zeta \lambda_{k}}\left[\sum_{j \neq i} \psi_{k, i} \psi_{k, j}\right] .
$$

Dividing by the positive constant $e^{\zeta \lambda_{1}} \sum_{j \neq i} \psi_{1, j}$ and taking the limit as $\zeta \rightarrow \infty$, the second part of the right-hand side vanishes, and we obtain again the eigenvector centrality $\psi_{1, i}$ of node $i$.

Remark 4.3. A natural question is how rapidly the degree (for $\zeta \rightarrow 0$ ) and eigenvector (for $\zeta \rightarrow \infty$ ) centrality limits are approached if the number of nodes $n$ in the network goes to infinity. From the Taylor expansions (see, for example, (4.3)) we see that the degree limit is reached more slowly if the row sums of $A^{2}$ grow as $n \rightarrow \infty$. In this case, as $n$ increases, $\zeta$ must be taken smaller and smaller before the ranking reduces to the one given by the degree. On the other hand, if the network grows in such a way that the maximum degree of any node remains uniformly bounded, then the rate of convergence is independent of the number $n$ of nodes, at least asymptotically.

The rate of convergence to the eigenvector centrality ranking is largely determined by the spectral gap, $\lambda_{1}-\lambda_{2}$. If the gap remains bounded below by a positive constant as $n \rightarrow \infty$, the value of $\zeta$ necessary to reach the eigenvector centrality limit is easily seen to grow at most like $O(\ln n)$, and in practice the rate of convergence is scarcely affected by the size of the network. If, on the other hand, the gap closes as $n \rightarrow \infty$, then the rate of convergence to the eigenvector centrality will become arbitrarily slow. The faster the gap closes for $n \rightarrow \infty$, the more rapidly the rate of convergence deteriorates.

We conclude this section with some comments on the measures $\mathscr{R}_{i}, \mathscr{C}_{i}$, and $\mathscr{T}_{i}$. While they all display the same limiting behavior and provide identical rankings in the small and 
large $\zeta$ limits, they provide different insights into the network structure (and therefore into node risk). For instance, it is well known that subgraph centrality (which is the same as circulability; see $[32,29]$ ) can discriminate between the nodes of certain regular graphs, that is, graphs in which all the nodes have the same degree. The same holds for transmissibility. Total communicability, on the other hand, cannot discriminate between the nodes of regular graphs (nor can degree and eigenvector centrality, of course). These measures are also different from a computational viewpoint. One advantage of the risk centrality based on total communicability is that it only requires the computation of the action of the matrix exponential $e^{\zeta A}$ on the vector $\overrightarrow{1}$. The entries of the resulting vector can be computed efficiently without having to compute any entry of $e^{\zeta A}$; see [10]. Modern Krylov-type iterative methods (like those based on the Lanczos or Arnoldi process) can handle huge networks (with many millions of nodes) without any difficulty. In contrast, the computation of the circulability requires the explicit computation of the diagonal entries of $e^{\zeta A}$ (the node transmissibility is then easily obtained by subtracting the circulability from the total communicability). Although there are techniques that can handle fairly large graphs (see [9]), these calculations are much more expensive than those for the total communicability. This limits the size of the networks that they can be applied to. However, for most financial networks the computation of the circulability is still feasible.

A final consideration pertains to the values assumed by the external risk parameter $\zeta$. Although, in principle, it can vary between 0 and infinity, for the purposes of most of the applications that follow, it may be sufficient to vary $\zeta$ between 0 and 1 . The rationale for using the interval $[0,1]$ relies on the fact that, at $\zeta=1$, the rankings given by $\mathscr{R}_{i}$ are already stabilizing around those provided by eigenvector centrality, and therefore no more interlacings between rankings are possible. As we will show, we typically observe a single point of interlacement, and it usually occurs before reaching the value $\zeta=1$. Furthermore, this choice is equivalent to fixing $t=1$ in the epidemic model solution (3.17), and, already as $\zeta$ approaches 1 , all the probabilities involved in that model become completely negligible or equal to 1 .

5. Risk-dependent centrality on a random network. For the analysis of real-world (financial and economic) networks it is necessary to investigate how informative the results obtained are with respect to the real system under analysis. This significance is typically addressed by comparing the results to those properties obtained from network null models. As such null models we consider here Erdős-Rényi (ER) random networks $\Gamma_{E R}(n, p)$ with $n$ nodes and wiring probability $p$ (see $[27,28]$ ), for which, in this section, we provide a series of analytical results. We start by generating a family of simulated ER graphs and discarding simulations for which the obtained graph is not connected.

In particular, we aim at testing how the external risk $\zeta$ and the probability $p$ and hence the graph density $\delta$ affect the results. For this purpose, we generate 1000 graphs $\Gamma_{E R}(n ; p)$ with $n=100$ at different values of $p$. For each graph, we compute the main measures for alternative values of $\zeta$. First, we report in Figure 3 the behavior of risk-dependent centrality $\mathscr{R}_{i}$, circulability $\mathscr{C}_{i}$, and transmissibility $\mathscr{T}_{i}$ as functions of the density, assuming a fixed high level of external risk, $\zeta=1$. Since the values of $\mathscr{R}_{i}$ are significantly increasing when the density of the graph increases, we display, in Figure 3(a), the distributions of the ratio between the risk-dependent centrality of each node $\mathscr{R}_{i}$ and its average value $\mathbb{E}\left(\mathscr{R}_{i}\right)$.

Copyright (c) by SIAM. Unauthorized reproduction of this article is prohibited. 
As might be expected, the centralities of nodes tend to be similar when $\delta \rightarrow 1$ and we move towards the complete graph, i.e., we observe a lower variability of the distribution of the ratios. Similar behaviors are also observed for $\mathscr{C}_{i}$ and $\mathscr{T}_{i}$, with a higher volatility for the circulability (see Figure 3(b) and Figure 3(c)).

In Figure 3(d), we show the distributions of the incidence of the circulability $\mathscr{C}_{i}$ on the risk-dependent centrality $\mathscr{R}_{i}$, that is, the distribution of the ratio $\frac{\mathscr{C}_{i}}{\mathscr{R}_{i}}$ again as a function of the density $\delta$. When $\zeta=1$, for all the graphs analyzed, the average value is around $\frac{1}{n}$, implying that the transmissibility has an average incidence of $\frac{n-1}{n}$ on $\mathscr{R}_{i}$. It is worthwhile to look at the variability of the distributions. When the density is extremely low, i.e., we refer to a very sparse graph, the heterogeneity of the node's degree affects the ratio $\frac{\mathscr{C}_{i}}{\mathscr{R}_{i}}$. For instance, when $\delta=0.1$, the circulability of a node ranges approximately from $0.15 \%$ to $2.5 \%$ of the riskdependent centrality for the same node. A lower variability is observed for higher densities. For instance, for $\delta=0.5$, the ratio $\frac{\mathscr{C}_{i}}{\mathscr{R}_{i}}$ varies between $0.6 \%$ and $1.3 \%$. For $\delta=0.95$, we observe a ratio between $0.9 \%$ and $1.15 \%$.

In Figure 4, we show the corresponding behaviors of risk-dependent centrality $\mathscr{R}_{i}$, circulability $\mathscr{C}_{i}$, and transmissibility $\mathscr{T}_{i}$ as functions of the density, but assuming a fixed low level of external risk, $\zeta=0.1$. Again all figures are based on 1000 randomly generated ER networks $\Gamma_{E R}(n ; p)$ with $\delta$ varying between 0.1 and 0.9 .

Focusing on the risk-dependent centrality ratio $\frac{\mathscr{R}_{i}}{\mathbb{E}\left(\mathscr{R}_{i}\right)}$, we observe that the standard deviation between nodes is lower in the low-risk framework $(\zeta=0.1)$ than in the high-risk one $(\zeta=1)$. For instance, when the density is equal to 0.1 , the standard deviation of the ratio moves from 0.20 for $\zeta=0.1$ to 0.37 for $\zeta=1$. At a phenomenological level, this behavior can be justified by the fact that differences between nodes tend to be enhanced when the network is highly risk-exposed.

Furthermore, the pattern of $\frac{\mathscr{C}_{i}}{\mathbb{E}\left(\mathscr{C}_{i}\right)}$ for $\zeta=0.1$ is very peculiar. In this case, when the network is very sparse, nodes show a similar circulability, while higher differences are observed when the density is around 0.5 .

Lastly, in Figure 5, we focus on the ratio $\frac{\mathscr{C}_{i}}{\mathscr{R}_{i}}$, and we report the incidence of the circulability on the risk-dependent centrality as a function of the external risk $\zeta$. In cases of sparse networks (Figure 5(a)), when the external risk is low, we have that the infection remains in larger part circulating in a loopy way around the nodes, while only a lower proportion of risk tends to be transmitted to other nodes. This is due to the fact that, for $A$ sparse and $\zeta$ small, the matrix $e^{\zeta A}=I+\zeta A+\frac{\zeta^{2}}{2} A^{2}+O\left(\zeta^{3}\right)$ is strongly diagonally dominant. When the external risk is high, as already observed, we have an average incidence of the circulability $\mathscr{C}_{i}$ on the risk-dependent centrality around $\frac{1}{n}$. On the contrary, when a very dense network is considered, the ratio $\frac{\mathscr{C}_{i}}{\mathscr{R}_{i}}$ is affected very little by the external risk. In this case, both $\mathscr{C}_{i}$ and $\mathscr{R}_{i}$ increase on average at the same rate when $\zeta$ increases. However, the decreasing behavior of $\frac{\mathscr{C}_{i}}{\mathscr{R}_{i}}$ is noticeable for very low values of $\zeta$.

In what follows we provide an exhaustive proof of the behaviors observed so far. Let us start with the pattern of the ratio $\frac{\mathscr{C}_{i}}{\mathscr{R}_{i}}$ at high density (see Figures $3(\mathrm{~d}), 4(\mathrm{~d})$, and $5(\mathrm{~b})$ ).

The asymptotic behavior of this ratio can be explained as a consequence of Theorem A.1 in the appendix, where we derive the close expressions of the three risk-dependent centrality measures for a complete graph. In fact, as $\delta \rightarrow 1$, the ER network approaches a complete 


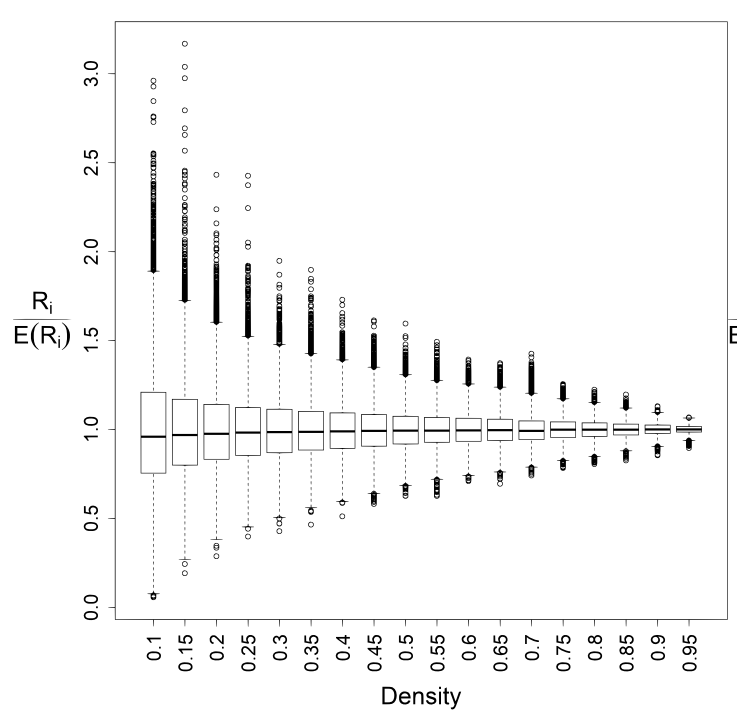

(a)

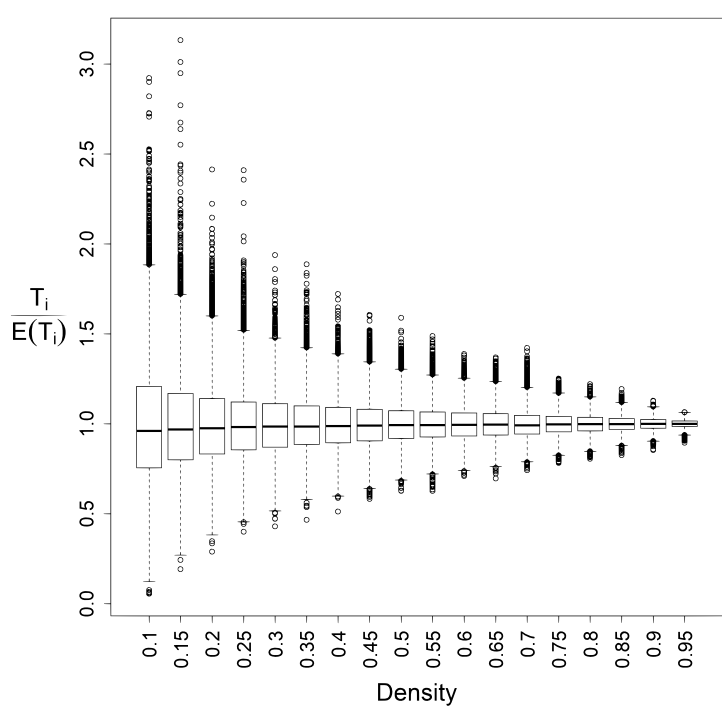

(c)

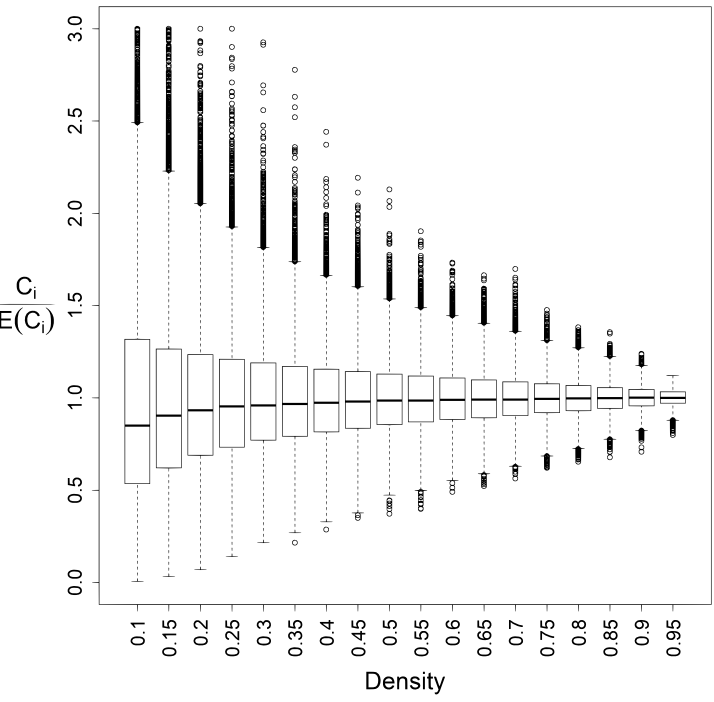

(b)

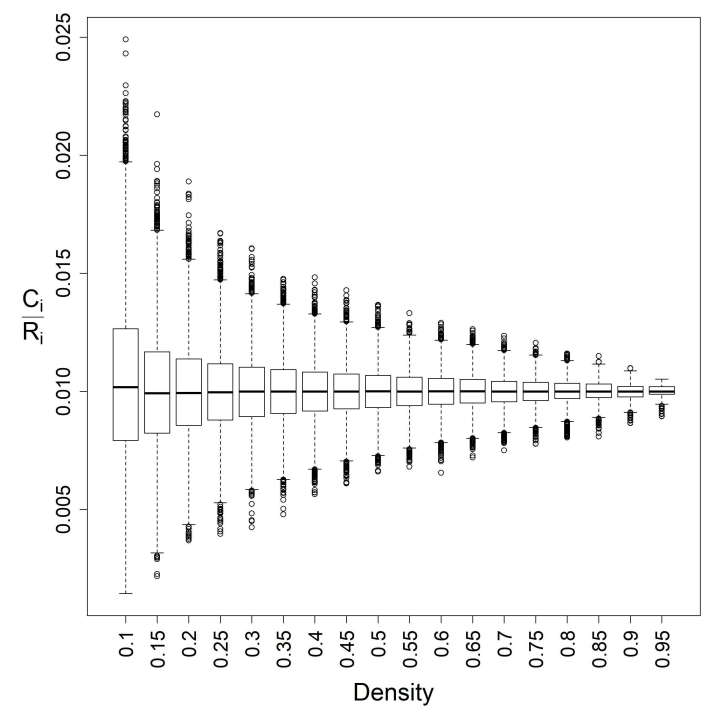

(d)

Figure 3. Figure (a) displays the distributions of the ratios between the risk-dependent centrality of each node $\mathscr{R}_{i}$ and the average risk-dependent centrality $\mathbb{E}\left(\mathscr{R}_{i}\right)$, computed assuming $\zeta=1$. Figures (b) and (c) display the analogous distributions for circulability and transmissibility. Figure (d) shows the distributions of the ratios between the circulability $\mathscr{C}_{i}$ and the risk-dependent centrality of each node $\mathscr{R}_{i}$, computed assuming $\zeta=1$. All figures are based on 1000 randomly generated $E R$ networks $\Gamma_{E R}(n ; p)$ with a density varying between 0.1 and 0.9 .

network, and, for $\zeta$ increasing, the ratio $\frac{\mathscr{C}_{i}}{\mathscr{R}_{i}}$ approaches $1 / n$, as shown in (A.1).

Nonetheless, this result can be generalized. In fact, for an ER network which is dense enough, the following property holds for any $\zeta$.

Copyright (C) by SIAM. Unauthorized reproduction of this article is prohibited. 


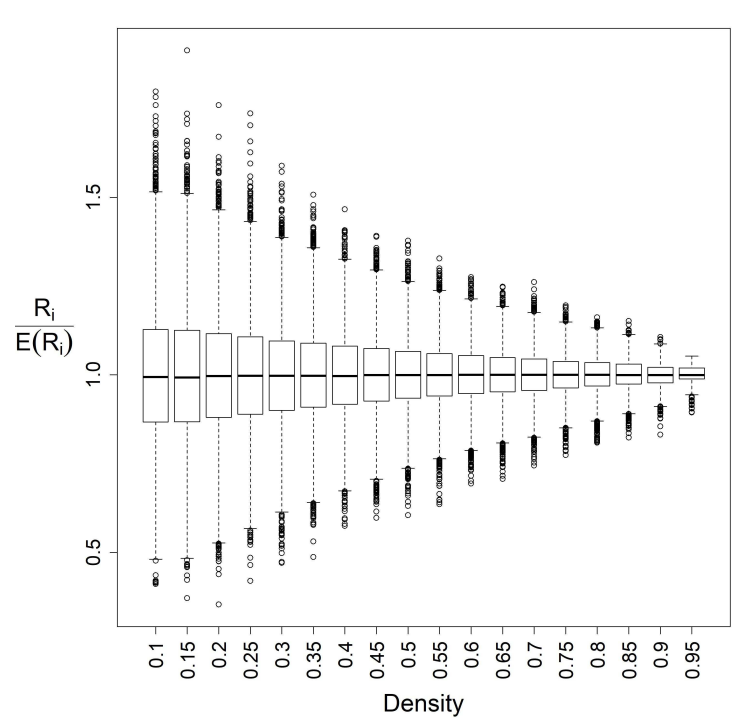

(a)

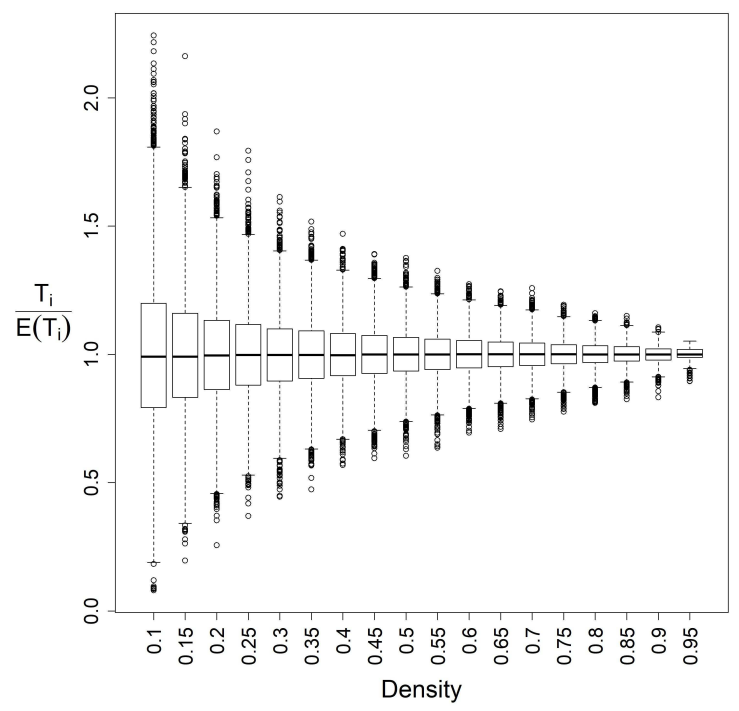

(c)

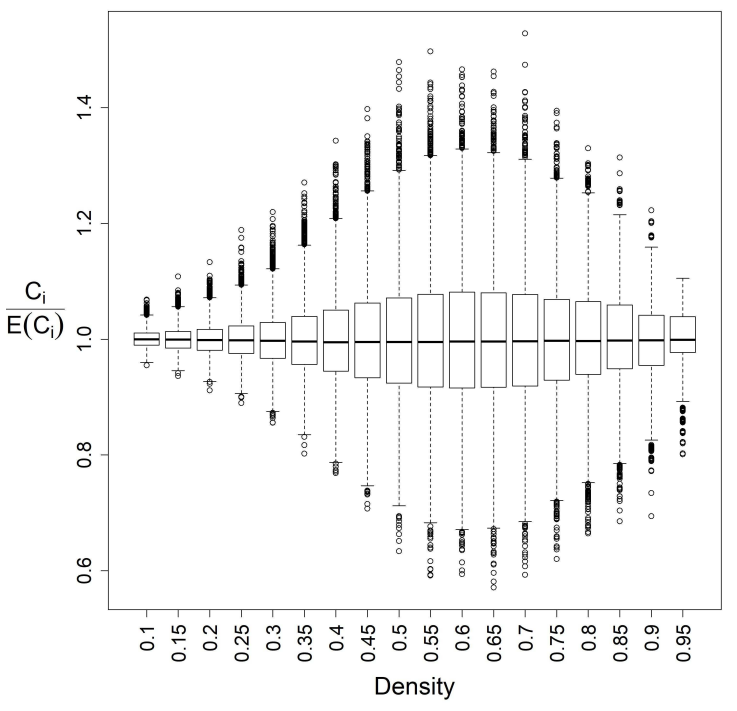

(b)

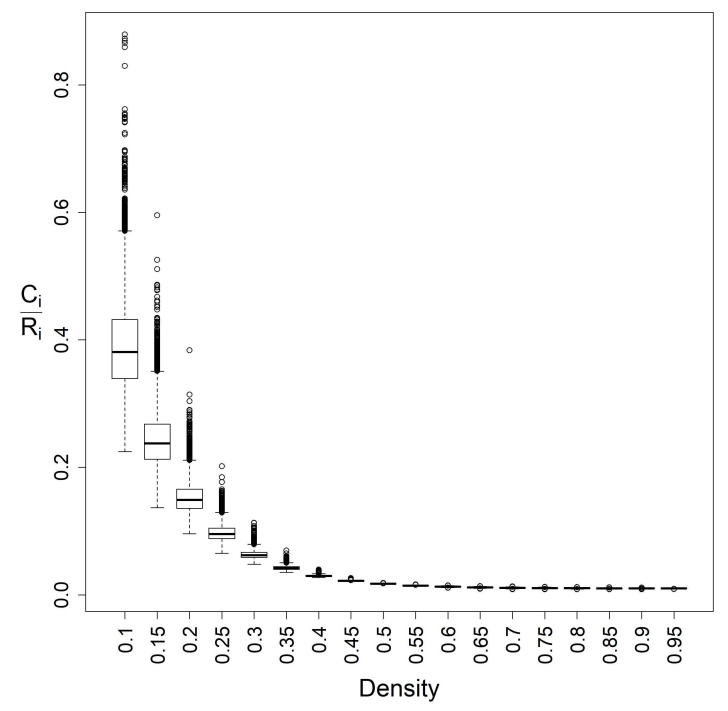

(d)

Figure 4. Figures (a), (b), (c), and (d) display the distributions of ratios $\frac{\mathscr{R}_{i}}{\mathbb{E}\left(\mathscr{R}_{i}\right)}, \frac{\mathscr{C}_{i}}{\mathbb{E}\left(\mathscr{C}_{i}\right)}, \frac{\mathscr{T}_{i}}{\mathbb{E}\left(\mathscr{T}_{i}\right)}$, and $\frac{\mathscr{C}_{i}}{\mathscr{R}_{i}}$, respectively, computed in case of a low external risk $(\zeta=0.1)$. All figures are based on 1000 randomly generated ER networks $\Gamma_{E R}(n ; p)$ with a density varying between 0.1 and 0.9 .

Theorem 5.1. Let $\Gamma_{E R}(n ; p)$ be an ER random graph with $n$ nodes and probability $p$. If the edge density of the graph is $\delta>(\log n)^{6} / n$ and $p(1-p)>(\log n)^{4} / n$, then for any node $i$

$$
\lim _{n \rightarrow \infty} \frac{n \mathscr{C}_{i}}{\mathscr{R}_{i}}=1
$$




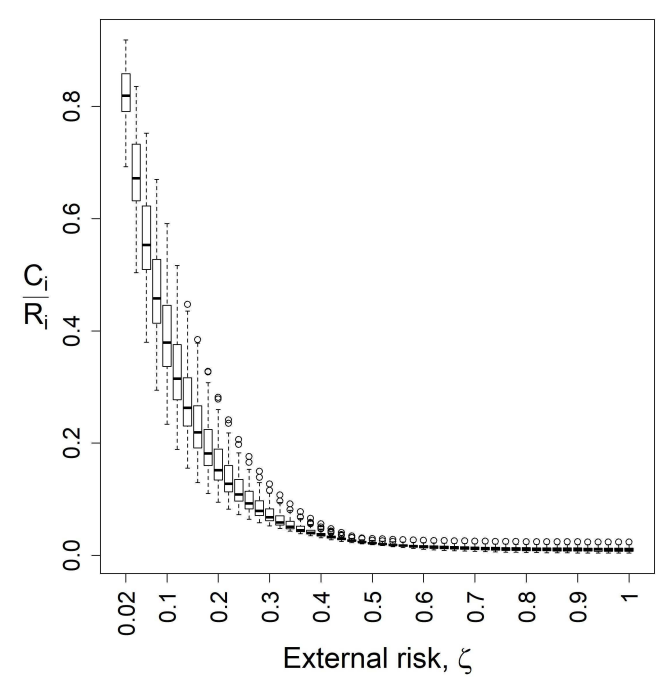

(a)

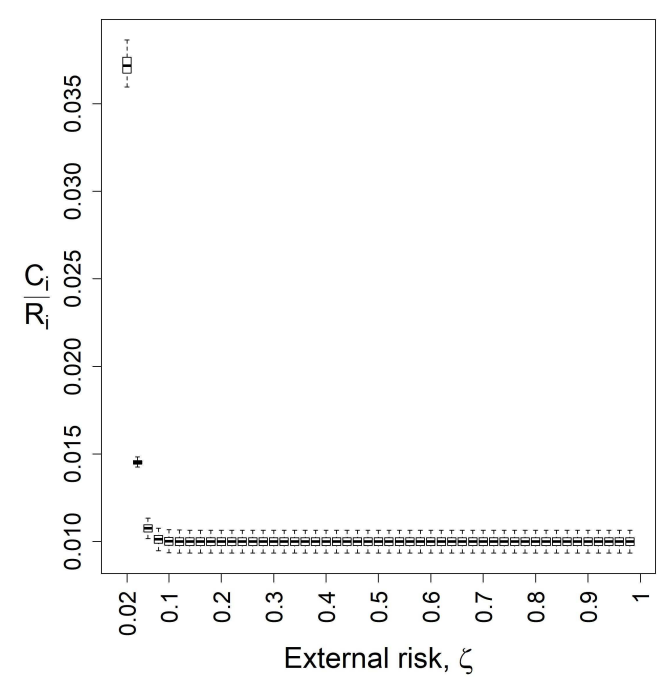

(b)

Figure 5. Figures report the distribution of the ratios between the circulability $\mathscr{C}_{i}$ and the risk-dependent centrality of each node $\mathscr{R}_{i}$, computed for different $\zeta$ and by using generated ER graphs with a density equal to 0.1 (Figure (a)) and 0.9 (Figure (b)), respectively. Both figures are based on 1000 randomly generated ER networks $\Gamma_{E R}(n ; p)$.

independently of $\zeta$.

Proof. Let us consider as usual that $\lambda_{1}>\lambda_{2} \geq \cdots \geq \lambda_{n}$ in a connected graph. It is known that in an ER graph the spectral gap $\left(\lambda_{1}-\lambda_{2}\right) \gg 0$. Indeed, as proved in [51], $\lim _{n \rightarrow \infty} \frac{\lambda_{1}}{n p}=1$, while $\lambda_{2}$ and $\lambda_{n}$ grow more slowly as $\lim _{n \rightarrow \infty} \frac{\lambda_{2}}{n^{\varepsilon}}=0$ and $\lim _{n \rightarrow \infty} \frac{\lambda_{n}}{n^{\varepsilon}}=0$ for every $\varepsilon>0.5$, respectively.

Then, if $n p(1-p)>(\log n)^{4}$, all but the largest eigenvalue lie with high probability in the interval $\sqrt{n p(1-p)}[-2+o(1),+2+o(1)]$ (see [82] and [57]). Therefore,

$$
\lim _{n \rightarrow \infty} \frac{\mathscr{C}_{i}}{\mathscr{R}_{i}}=\lim _{n \rightarrow \infty} \frac{\psi_{1, i}^{2} e^{\zeta \lambda_{1}}+\sum_{k=2}^{n} \psi_{k, i}^{2} e^{\zeta \lambda_{k}}}{\psi_{1, i}\left(\vec{\psi}_{1}^{T} \overrightarrow{1}\right) e^{\zeta \lambda_{1}}+\sum_{k=2}^{n} \psi_{k, i}\left(\vec{\psi}_{k}^{T} \overrightarrow{1}\right) e^{\zeta \lambda_{k}}}=\frac{\psi_{1, i}}{\sum_{j=1}^{n} \psi_{1, j}} .
$$

The edge density of an ER graph is $\delta=p$. In [26], it was proved that for $n p>(\log n)^{6}$, there exists a positive constant $C$ such that the following inequality holds:

$$
\left\|\vec{\psi}_{1}-\frac{1}{\sqrt{n}} \overrightarrow{1}\right\|_{\infty}<C \frac{1}{\sqrt{n}} \frac{\log n}{\log (n p)} \sqrt{\frac{\log n}{n p}},
$$

which in plain words means that an ER graph of density $\delta>(\log n)^{6} / n$ is "almost" regular when $n \rightarrow \infty$. That is, $\lim _{n \rightarrow \infty} \sqrt{n} \psi_{1, i}=1$ for every node $i$. Thus, the result immediately follows.

It is worth pointing out that, when the density of an ER network is very low, the standard deviation of the ratio $\frac{\mathscr{C}_{i}}{\mathscr{R}_{i}}$ is very large with respect to that of ER networks with large densities 
(as shown in Figure 3(d)). As we have proved before, the convergence of this ratio to the value $n^{-1}$ takes place only when the density of the graph is relatively large. Let us now analyze what happens when the edge density is very small for large graphs. In this case, we observe a slower decay of the ratio $\frac{\mathscr{C}_{i}}{\mathscr{R}_{i}}$ as a function of the external risk in the range $[0,1]$ (see Figure 5(a)). This fact can be easily proven as follows. In general, both the numerator and denominator of this ratio can be expressed as infinite series of the type

$$
\begin{gathered}
\mathscr{C}(\zeta)_{i}=Q(\zeta)=1+a_{2} \zeta^{2}+\cdots+a_{k} \zeta^{k}+\cdots, \\
\mathscr{R}(\zeta)_{i}=H(\zeta)=1+b_{1} \zeta+\left(a_{2}+b_{2}\right) \zeta^{2}+\cdots+\left(a_{k}+b_{k}\right) \zeta^{k}+\cdots=Q(\zeta)+L(\zeta),
\end{gathered}
$$

where $a_{k}$ counts the number of closed walks of length $k$ starting and ending at node $i$ and $b_{k}$ counts all the open walks of length $k$ starting at $i$ and ending at any node $j \neq i$. Let us consider

$$
\begin{aligned}
& \frac{d}{d \zeta}\left(\frac{Q(\zeta)}{Q(\zeta)+L(\zeta)}\right)=\frac{L(\zeta) Q^{\prime}(\zeta)-L^{\prime}(\zeta) Q(\zeta)}{[Q(\zeta)+L(\zeta)]^{2}} \\
& =\frac{\left(2 a_{2} b_{1} \zeta^{2}+\cdots+2 a_{2} b_{k} \zeta^{k+1}+\cdots\right)-\left(b_{1}+2 b_{2} \zeta+a_{2} b_{1} \zeta^{2}+\cdots+b_{1} a_{k} \zeta^{k}+\cdots\right)}{[Q(\zeta)+L(\zeta)]^{2}} .
\end{aligned}
$$

Then, for certain $\zeta<1$ the numerator of the previous expression is negative, which means that the ratio $\frac{\mathscr{C}_{i}(\zeta)}{\mathscr{R}_{i}(\zeta)}$ is monotonically decreasing with $\zeta$. For instance, let us make a second order approximation to the polynomials $Q(\zeta)$ and $H(\zeta)$. Then, we have

$$
\frac{Q(\zeta)}{H(\zeta)}=\frac{1+\frac{1}{2} \zeta^{2} k_{i}}{1+\zeta k_{i}+\frac{1}{2} \zeta^{2}\left(k_{i}+P_{2, i}\right)},
$$

where $P_{2, i}$ is the number of paths of length 2 (wedges) starting at node $i$. In an ER graph $\mathbb{E}\left(k_{i}\right)=(n-1) p$ and $\mathbb{E}\left(P_{2, i}\right)=(n-1)^{2} p^{2}-(n-1) p$. Thus,

$$
\frac{Q(\zeta)}{H(\zeta)} \approx \frac{1+\frac{1}{2} \zeta^{2}(n-1) p}{1+\zeta(n-1) p+\frac{1}{2} \zeta^{2}(n-1)^{2} p^{2}}=\frac{1+\frac{\bar{k}}{2} \zeta^{2}}{1+\bar{k} \zeta+\frac{\bar{k}^{2}}{2} \zeta^{2}},
$$

where $\bar{k}=(n-1) p$ is the mean degree. The first derivative of this rational function is

$$
\frac{d}{d \zeta}\left(\frac{Q(\zeta)}{H(\zeta)}\right)=\frac{2 \bar{k}^{2} \zeta^{2}-(4 \bar{k}(\bar{k}-1) \zeta+4 \bar{k})}{\left(2+2 \bar{k} \zeta+\bar{k}^{2} \zeta^{2}\right)^{2}}
$$

which is always negative for any $\bar{k} \geq 1$ and $0 \leq \zeta \leq 1$, as can be seen in Figure 6 . Moreover, the absolute value of this derivative increases as $\bar{k}$ decreases, implying a slower decay in the function $\frac{\mathscr{C}_{i}(\zeta)}{\mathscr{R}_{i}(\zeta)}$ for lower densities.

To conclude this section, we want to focus on the rankings produced by the two main centrality measures $\mathscr{R}_{i}$ and $\mathscr{C}_{i}$ and on the similarities between them. In particular, we are interested in determining if, or for what type of networks, the different centrality measures 


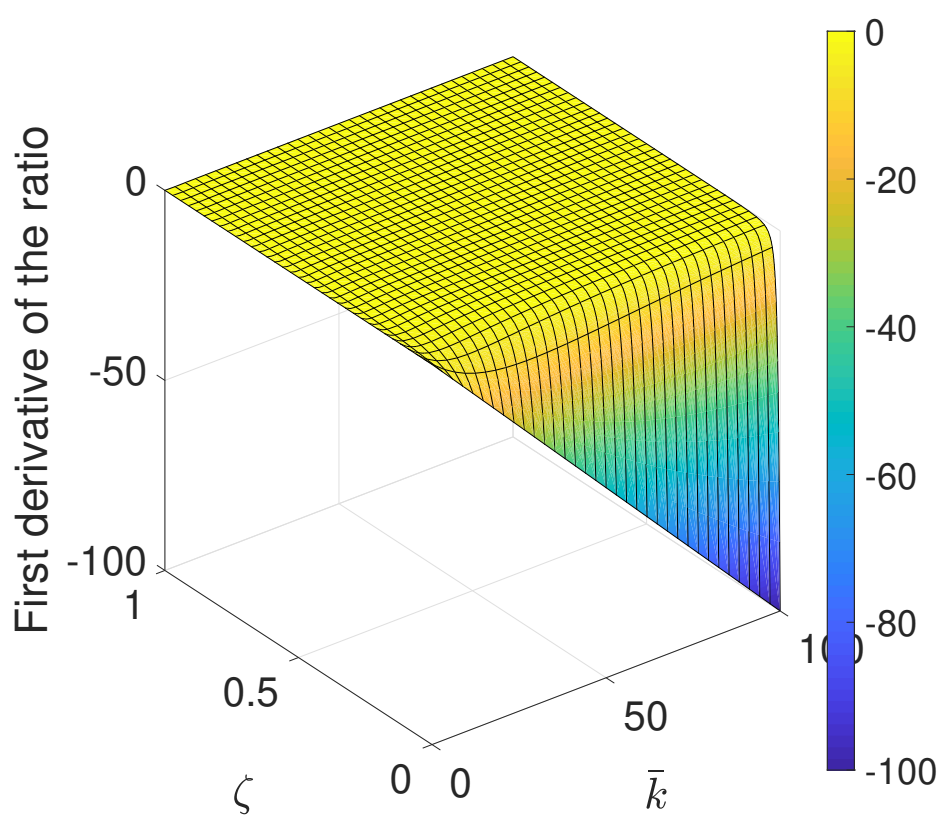

Figure 6. Illustration of the behavior of the derivative of the ratio $\frac{\mathscr{C}_{i}(\zeta)}{\mathscr{R}_{i}(\zeta)}$ for values of $0 \leq \zeta \leq 1$ and for the parameter $\bar{k} \geq 1$.

provide similar rankings. To this end, we display in Table 1 the Spearman correlation coefficient between the risk-dependent centrality $\mathscr{R}_{i}$ and the circulability $\mathscr{C}_{i}$ for different graph densities and for various values of $\zeta$. On average, we observe a strong positive monotonic dependence between the two centrality measures. As expected, the two measures tend towards the perfect monotonicity as the density arises. The behavior with respect to $\zeta$ is noteworthy. The higher dependence is observed in a low-risk framework $(\zeta=0.1)$, while a slight reduction is noticeable when higher risk contexts are analyzed, providing again empirical evidence of the fact that differences between nodes are increased in stressed conditions. Furthermore, this result is in line with the higher incidence of $\mathscr{C}_{i}$ on $\mathscr{R}_{i}$ as $\zeta$ vanishes, which was discussed previously. For the sake of brevity, we do not report the Spearman correlation between $\mathscr{R}_{i}$ and $\mathscr{T}_{i}$. However, in all cases, the coefficient is larger than 0.9999 .

Table 1

Spearman correlation coefficients between $\mathscr{C}_{i}$ and $\mathscr{R}_{i}$ in ER graphs with 100 vertices at different densities and different values of $\zeta$.

\begin{tabular}{|cc|ccccc|}
\hline & & \multicolumn{5}{|c|}{ Density } \\
& & 0.1 & 0.3 & 0.5 & 0.7 & 0.9 \\
\hline \multirow{4}{*}{$\zeta$} & 0.1 & 0.9947 & 0.9967 & 0.9971 & 0.9994 & 0.9998 \\
& 0.5 & 0.9844 & 0.9950 & 0.9966 & 0.9994 & 0.9998 \\
& 1.0 & 0.9813 & 0.9950 & 0.9966 & 0.9994 & 0.9998 \\
\hline
\end{tabular}

6. Analysis of real-world financial networks. In this section, we perform some empirical studies in order to assess the effectiveness of the proposed approaches. We consider two 
different networks. In the first one, we collected daily returns of a dataset referring to the time period ranging from January 2001 to December 2017 that includes 102 leading U.S. stock constituents of the S\&P 100 index at the end of 2017. Data have been downloaded from Bloomberg. Returns have been split by using monthly stepped six-month windows. This means that the data of the first in-sample window of width six months are used to build the first network. The process is repeated, rolling the window one month forward until the end of the dataset is reached, obtaining a total of 199 networks. The first network, denoted as "1-2001" covers the period 1st of January 2001 to 30th of June 2001. The latter one ("7-2017") covers the period 1st of July 2017 to 31st of December 2017.

Hence, for each window, we have a network $\Gamma_{t}=\left(V_{t}, E_{t}\right)$ (with $\left.t=1, \ldots, 199\right)$, where assets are nodes and links are weighted by computing the correlation coefficient ${ }_{t} \rho_{i, j}$ between the empirical returns of each couple of assets. Notice that the number of assets can vary over time. Indeed, as mentioned, we have considered the 102 asset constituents of the S\&P 100 index at the end of 2017. Some of these assets have no information available for some specific time periods. Therefore, in each window, we have considered only assets whose observations are sufficiently large to ensure a significant estimation of the correlation coefficient. However, it is not the aim of this paper to deal with the effects of alternative estimation methods. As a consequence, the number of nodes in the 199 networks varies from 83 to 102 during the time period.

Then, we follow the methodology proposed in $[65,72]$, and we use the nonlinear transformation, based on distances ${ }_{t} d_{i, j}:{ }_{t} d_{i, j}=\sqrt{2\left(1-{ }_{t} \rho_{i, j}\right)}$. The distance matrix $\left.D_{t}={ }_{t} d_{i, j}\right]_{i, j \in V_{t}}$, with elements $0 \leq{ }_{t} d_{i, j} \leq 2$, becomes the weighted adjacency matrix of the graph $\Gamma_{t}$. As proposed in [72], we extract the minimum spanning tree $T_{t}$. This is a simple connected graph that connects all $n_{t}$ nodes of the graph with $n_{t}-1$ edges such that the sum of all edge weights $\sum_{t} d_{i, j} \in T_{t} t d_{i, j}$ is minimum. As shown in [72], this minimum spanning tree, as a strongly reduced representative of the whole correlation matrix, bears the essential information about asset correlations. Furthermore, the study of the centrality of nodes and the analysis of the evolution of the tree over time are two critical issues in portfolio selection problems (see $[72,75,77])$.

The second dataset consists of a network of the top corporates in the U.S. in 1999 according to Forbes magazine. The network is constructed as follows. First we consider a bipartite network in which one set of nodes consists of companies and the other consists of Chief Executive Officers (CEOs) of such companies. As one CEO can be in more than one company, we make a projection of this bipartite graph into the company-company space. In this way, the nodes represent corporations, and two corporations are joined by an edge if they share at least one director. We consider two versions of this network. In the first we use the number of directors shared by two companies as an edge weight, and in the second we use the binary version of the first. We will refer to these as the weighted and the binary network, respectively. The network has 824 nodes, made up of one giant component of 814 nodes. We selected the giant component, with its binary and weighted adjacency matrices. For a comprehensive description of this network see, for instance, [20].

Networks, derived by both datasets, have been studied by computing the total communicability, circulability, and transmissibility for each node with $\zeta$ varying in $(0,1]$ with step 0.01 .

Copyright (c) by SIAM. Unauthorized reproduction of this article is prohibited. 
6.1. Network of assets. Starting from the asset trees $T_{t}$, we measure the relevance of each node by using the risk-dependent centrality $\mathscr{R}_{i}$ and by testing different values of $\zeta$. We consider in Figure 7 the rankings' distributions of each asset. Different outcomes of each distribution have been obtained by computing the rankings based on $\mathscr{R}_{i}$ for alternative values of $\zeta$ in the interval $(0,1]$ with step 0.01 . These results pertain to the first network 1-2001, namely, the network based on data that cover the period 1st of January 2001 to 30th of June 2001. We observe that some nodes show a significant variability according to different values of $\zeta$. Indeed, some assets have climbed more than 20 positions in the ranking when $\zeta$ increases. For instance, Amazon (node 7 in Figure 7) moved from position 66 to 41 in case of low and high risk, respectively. Conversely, Exelon Group (node 32 in Figure 7) lowered its ranking from 15 to 46 . On the other hand, the most central nodes in the network remain very central also when external risk is very high. We have indeed that a position within the top 6 is quite stable for different values of $\zeta$. Top assets only change position a bit, preserving their central role. For instance, United Technologies Corporation (node number 79 in Figure 7) is at the top of the ranking, independent of $\zeta$.

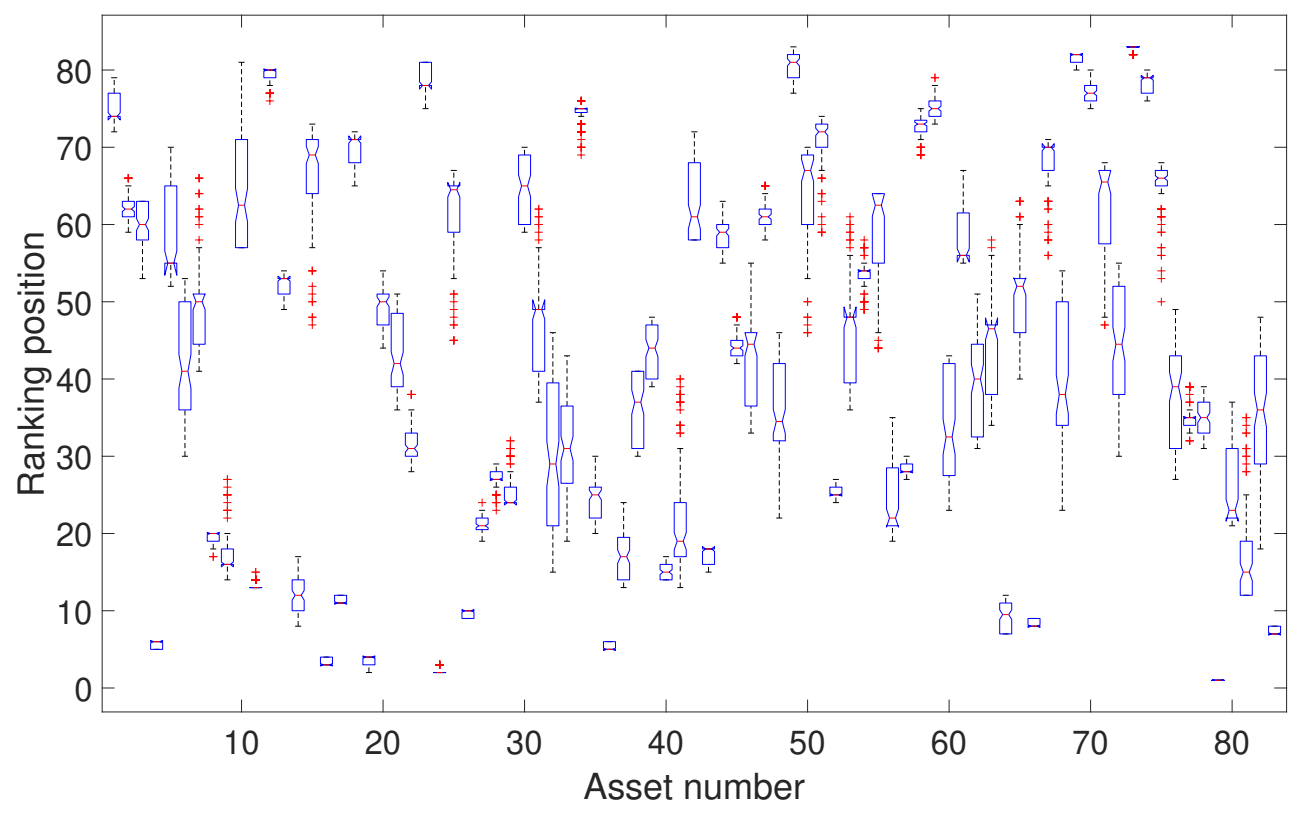

Figure 7. Figure reports the distribution of nodes' rankings based on $\mathscr{R}_{i}$ with respect to $\zeta$. For each distribution, the set of outcomes is given by the rankings of $\mathscr{R}_{i}$ computed for alternative values of $\zeta$. Results pertain to the network $T_{1}$, i.e., the asset-tree in the first window 1-2001.

If we consider the period of the global financial crisis of 2007-2008 (see Figure 9 and Figure 10), we observe an increase in the rankings' volatility. In shock periods, centrality of nodes is more affected by the value of $\zeta$. In particular, to catch rankings' volatility, we report in Figure 8 the standard deviations of rankings of each asset computed varying $\zeta$. In shock periods, results confirm higher average volatility as well as positive skewed distributions because of a greater number of assets whose ranking is highly affected by the value of $\zeta$. We 
also tested that differences in average volatility are significant by means of a paired $t$-test, useful for comparing the same sample of assets at different time periods. When the network 1-2001 is compared with the two networks covering the period of crisis (End 2007 or End 2008), we obtain $p$-values around $10^{-5}$ and $10^{-8}$ that confirm strong evidence against the null hypothesis that the average difference between the two samples is zero. As expected, the test is not statistically significant ( $p$-value is 0.31 ) when networks covering the period of the global financial crisis are compared.

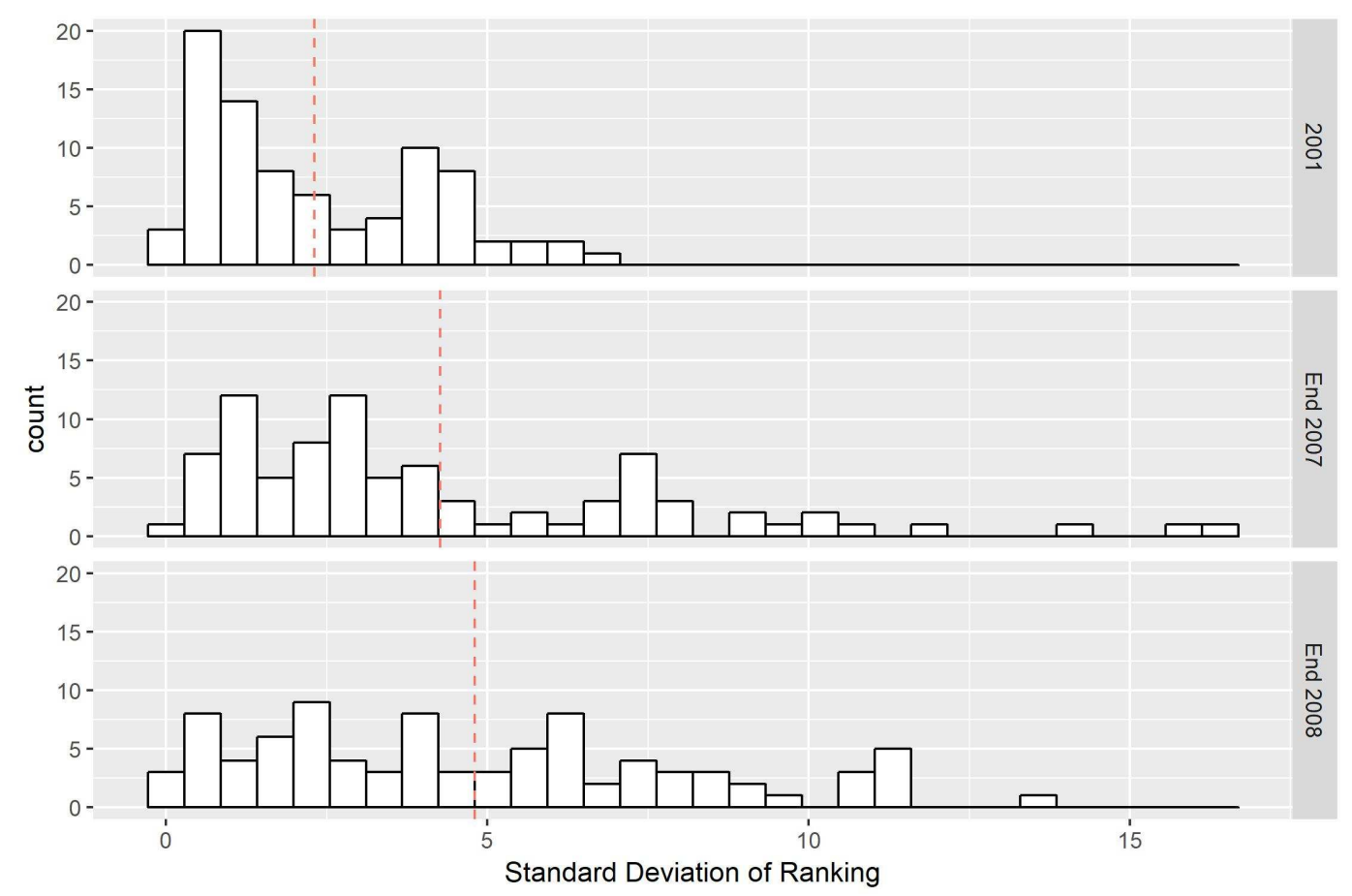

Figure 8. Figure reports the distribution of standard deviations of nodes' rankings based on $\mathscr{R}_{i}$ with respect to $\zeta$. For each distribution, the set of outcomes is given by the standard deviation of rankings of $\mathscr{R}_{i}$ computed for alternative values of $\zeta$. Results pertain to, respectively, the network in the first window 1-2001, at the end of 2007, and at the end of 2008. The dotted red lines indicate the average standard deviation: Values are equal to $2.31,4.27$, and 4.80 , respectively.

Concerning the behavior of specific assets, we observe, for instance, that some assets move down by approximately 60 positions from a low risk to a high risk framework. Two examples are represented by Danaher Corporation and Honeywell International (assets 28 and 43, respectively, in Figure 9). Instead, Accenture PLC (node 3 in Figure 9) increased its ranking from position 61 to 11.

Even top central nodes are affected by $\zeta$ as the volatilities of their rankings show. The rankings confirm the relevance of United Technologies Corporation (node number 82 in Figure 9 and 83 in Figure 10), which is again at the top of the ranking at the end of 2017, independent of $\zeta$. At the end of 2008, the centrality of this asset is also confirmed, although a bit of variability in the ranking is observed for this firm. 


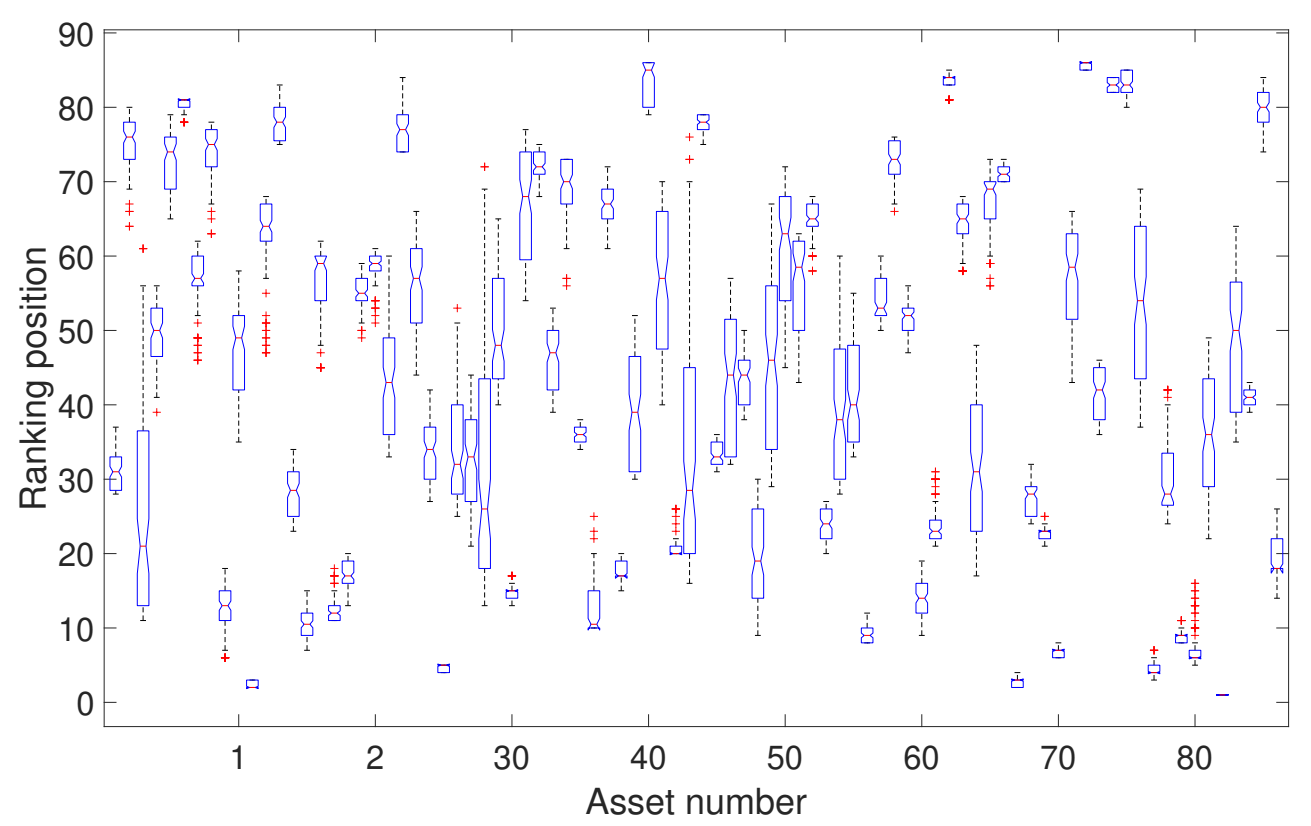

Figure 9. Figure reports the distribution of nodes' rankings based on $\mathscr{R}_{i}$ with respect to $\zeta$. For each distribution, the set of outcomes is given by the rankings of $\mathscr{R}_{i}$ computed for alternative values of $\zeta$. Results pertain to the asset-tree at the end of 2007.

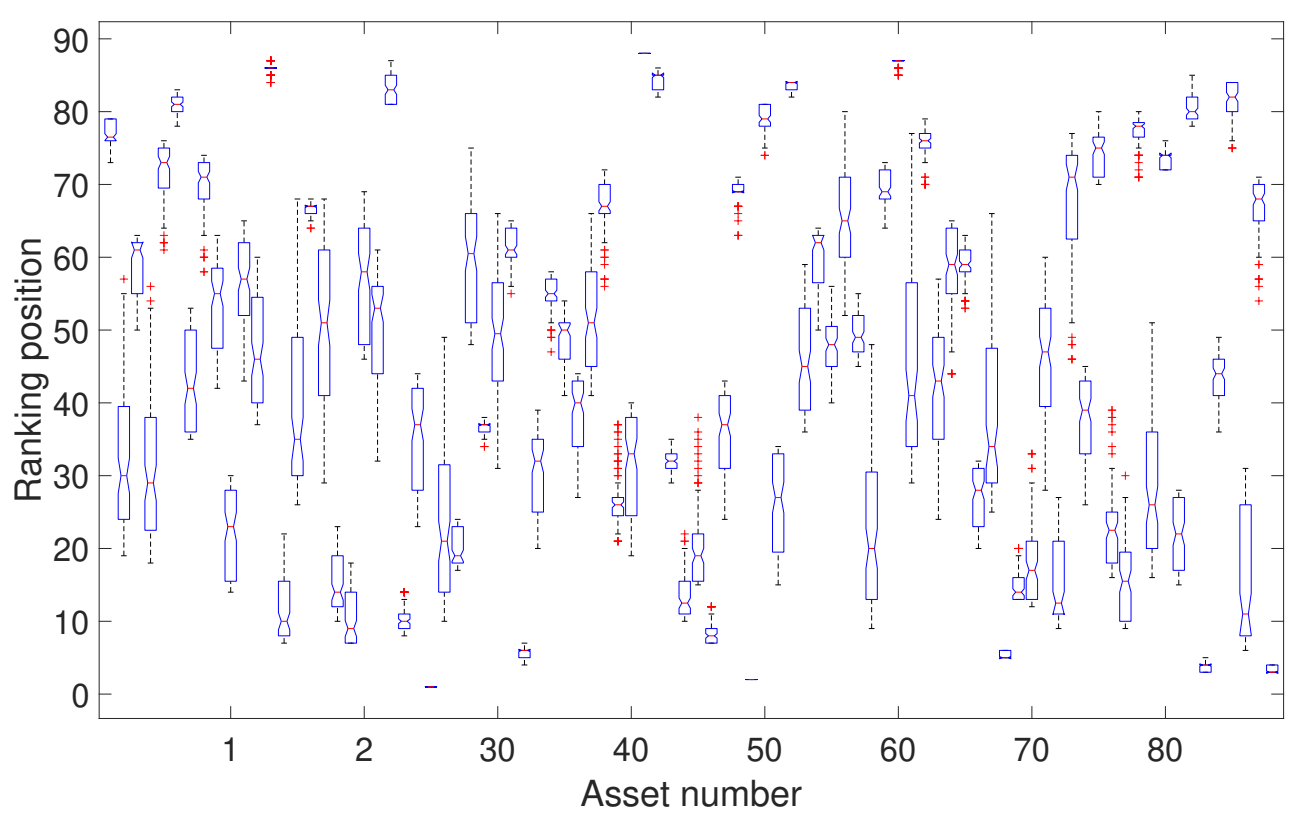

Figure 10. Figure reports the distribution of nodes' rankings based on $\mathscr{R}_{i}$ with respect to $\zeta$. For each distribution, the set of outcomes is given by the rankings of $\mathscr{R}_{i}$ computed for alternative values of $\zeta$. Results pertain to the asset-tree at the end of 2008.

Copyright $\odot$ by SIAM. Unauthorized reproduction of this article is prohibited. 
6.2. U.S. corporate network. We now analyze the network of U.S. top corporates in 1999 according to Forbes magazine. Before starting our analysis, let us explain the importance of studying spreading dynamics on this network. According to this network, the board of directors of a given corporation is formed by a few members, some of whom are also on the boards of other corporations. Then, such directors serving on more than one board can act as spreaders of information between the corresponding corporations. Such information can be about future (favorable or unfavorable) economic situations, alarms, market opportunities, or anything that could be of interest to the companies' directors. Due to the global connectivity of the system, such "information" can be spread across the whole network, "infecting" all the corporations in a relatively short time. As we have mentioned before, epidemiological models have also being used for modeling such propagation dynamics (see section 1.1.).

Hence, we devote this section to the investigation of whether a significant increase of the risk-dependent centrality is a proxy of the vulnerability of the corporate to financial infections propagating on the network. At first, we should note the fact that the network we are considering here was built based on data corresponding to year 1999. At this year the level of stress of the international economic system was relatively high due to the fact that the East Asian financial crisis occurred in the years 1997-1998, which was also followed by the Russian default of 1998. The two aforementioned financial crises had a ripple effect on the U.S. market. In the literature, for instance, the so-called "fire-sale" FDI (Foreign Direct Investment) phenomenon, that is, the surge of massive foreign acquisitions of domestic firms during a financial crisis [2, 24], is well documented. Thus, the level of stress and infectability of the system for the next few years after 1999 (we will eventually see that these correspond to the period 2000-2002) is expected to be significantly larger than in the subsequent years when the effects of these crises gradually relaxed. Therefore, we continue our analysis by considering that the level of infectability in 1999 is high, and we investigate the effects of relaxing such a condition to lower levels of stress. That is, we start by assuming that in 1999 the external market turmoil could be represented by a value of $\zeta=1$, and we want to find out how the companies change their ranking positions in term of risk-dependent centrality ${ }^{3} \mathscr{R}_{i}$ as $\zeta$ vanishes. To this purpose, we set up different initial conditions in the contagion model described by (3.10), assigning to each year a different value of the infectability parameter $\gamma$, according to the environmental conditions of the market. Therefore, we let $\zeta$ factors reduce year by year in order to reflect a reduction in the overall stress on the network. In particular, we decrease $\zeta$ linearly from 1 to 0 in the period 1999-2003. Therefore, rankings based on the risk-dependent centrality computed for $\zeta=1$ allow us to assess the relevance of each corporate in 1999. Lowering $\zeta$, we test how the positions of firms vary over time when the external risk reduces. It is noteworthy that the connection between this parameter and the risk could be quite loose, but as shown in the following analysis the model seems to work quite well at describing firms that reduce their SVC in the period.

The variation of rankings is then compared with the pattern of the shareholder value creation (SVC) over time. According to the OECD Principles of Corporate Governance,

\footnotetext{
${ }^{3}$ The analysis has been also developed for circulability and transmissibility, but, because of the significantly high rank correlation between $\mathscr{R}_{i}$ and $\mathscr{T}_{i}$ (with Spearman correlation coefficients larger than 0.99), we focus here only on $\mathscr{R}_{i}$.
} 
corporations should be run, first and foremost, in the interests of shareholders (OECD 1999). Therefore, companies should work to increase their shareholder values. Increasing shareholder value cannot be done without risk. It is known [61] that in the shareholder value model, companies usually take more risk than needed in order to maximize SVC. As a consequence of this additional risk, companies acquire debts which could make them unstable and more exposed to the risk of bankruptcy. Acquiring large debts is seen as conductive to increasing shareholder value, due to the potential of the company to increase value when it has started from a low baseline. Thus, there is a relation between SVC and risk, because in searching for large SVC the companies increase their risks to attract more investors and increase potential value gain, but, at the same time, the risk also puts the company in a more vulnerable position for bankruptcy and collapse.

To support our interpretation, we make use of SVCs of the companies ${ }^{4}$ in the S\&P 500 for the period 1999-2003 that have been collected by Fernandez and Reinoso (see [35]). Hence, we use SVC as a proxy for risk. Indeed, the global average of SVC reflects very well what happens for the period 1999-2003. After the financial crisis of 1998, the world was at a higher level of risk, which is reflected by a dramatic drop of the SVC in year 2000 from a positive value in 1999 to a negative one in 2000. This situation remained until 2002 but eventually recovered to positive in 2003 (see Figure 11). It is noteworthy that the data for SVC was reported by Fernandez and Reinoso for the years 1993-2003. From this long period we select the segment 1999-2003, which contains exactly the valley produced from the financial crisis of 1998, and also because the data used for building the corporate network is from 1999. That is, it corresponds to a segment of time in which the world economy dropped due to a crisis and then eventually recovered from it.

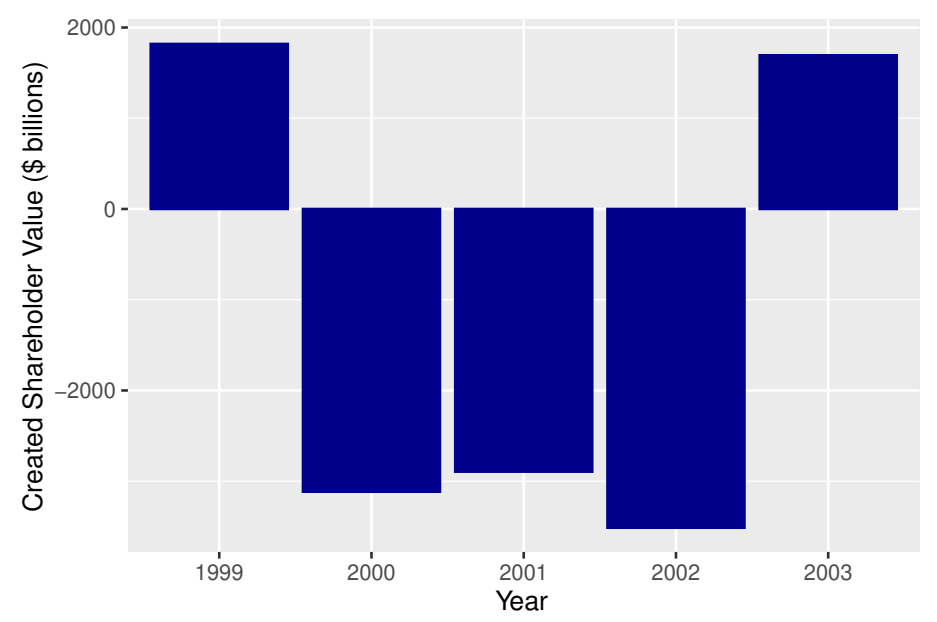

Figure 11. Total created shareholder value (\$ billion) of firms constituting the index SEP 500 for the period 1999-2003 (data taken from [35]).

We focus our statistical analysis on the predictability of the risk-dependent centrality

\footnotetext{
${ }^{4}$ In particular, we use a sample of 337 companies in our network whose SVCs are made available in the dataset available in [35].
}

Copyright (C) by SIAM. Unauthorized reproduction of this article is prohibited. 
on the evolution of the SVC. We consider the evolution of the SVC of a company for the period 1999-2003, which is the period immediately after the network of the corporate elite in the U.S. was built. As a proxy for the evolution of the SVC of a company we consider the Pearson correlation coefficient $\rho$ of the ranking position of the company based on SVC versus the reciprocal of the year. In this case, a negative (positive) value of $\rho$ indicates that the corresponding company decreases (increases) its SVC from 1999 to 2003 when the global external infectability decreases. Therefore, we apply a linear discriminant analysis (LDA) to classify the companies into two groups: (i) those with negative trend in the SVC for this period, and (ii) those with a positive one. The only predictor used for this classification is the parameter $\Delta \operatorname{Rank}\left(\mathscr{R}_{i}\right)$. This parameter is the difference between the ranking position of the company $i$ when $\zeta=1$ and the ranking position of the same company when $\zeta=0.01$. In other words, a negative (positive) value of $\Delta \operatorname{Rank}\left(\mathscr{R}_{i}\right)$ means that the company dropped (increased) its exposure to risk when the infectability of the system was lower.

Before proceeding with the application of the LDA on the whole sample at our disposal, we eliminate a few companies whose correlation coefficient between SVC and the reciprocal of the year is marginal (i.e., close to zero). We test empirically the effect produced by the removal of companies for which $|\rho|<a$ for different values of the threshold $a$, e.g., $a=0.01,0.025,0.05,0.075,0.1$. The best classification of the companies into the two groups analyzed is obtained by eliminating those companies for which $|\rho|<0.05$. In this case the total accuracy of the LDA model is $60.5 \%$. That is, 200 out of 332 of the companies are classified correctly in their respective groups representing their trends in shrinking SVC or expanding it. In particular, the fitted LDA model is $\hat{Y}_{i}=-0.3177+0.0102 \Delta \operatorname{Rank}\left(\mathscr{R}_{i}\right)$, where $\hat{Y}_{i}$ is the predicted response variable of our analysis that allows us to classify companies in their respective group. The positive coefficient of the variable $\Delta \operatorname{Rank}\left(\mathscr{R}_{i}\right)$ indicates that (i) increasing the exposure to risk $\left(\Delta \operatorname{Rank}\left(\mathscr{R}_{i}\right)>0\right)$ tends to expand the SVC of the company, and (ii) decreasing the exposure to risk $\left(\Delta \operatorname{Rank}\left(\mathscr{R}_{i}\right)<0\right)$ tends to shrink the SVC of the company. For both groups, we report in Figure 12(a) a comparison between the predicted value with the LDA and the observed value for each firm. Red squares below the line and blue circles over the line are well classified, while blue circles below the line and red squares over the line are wrongly classified. Furthermore, in Figure 12(b) we report the related confusion plot, where the number of true negative and true positive are on the antidiagonal (lower and upper parts, respectively) and the number of false negative and false positive are on the main diagonal (lower and upper parts, respectively). Notice the low classification performance of the model when only companies that expand their SVC are considered (in this regard, see in Figure 12(b) the companies that belong to the observed class denoted with the sign "+"). For instance, from 147 companies in the network which increase their SVC in the period 19992003 , only 36 are correctly predicted by $\Delta \operatorname{Rank}\left(\mathscr{R}_{i}\right)$ in their class. On the contrary, from the 175 companies that shrink their SVC in the period 1999-2003, the variable $\Delta \operatorname{Rank}\left(\mathscr{R}_{i}\right)$ correctly predicts 157 companies in this class. That is, the risk-dependent centrality of the companies clearly identifies about $90 \%$ of the companies which will shrink their SVC in the period 1999-2003, using only data referring to the year 1999. In plain words, our results indicate that diminishing the exposure to risk when the external conditions of infectability are low, with high probability, reduces the SVC of a company.

Let us conclude with the following remark. Even if "good" companies increase their risk-

Copyright (c) by SIAM. Unauthorized reproduction of this article is prohibited. 


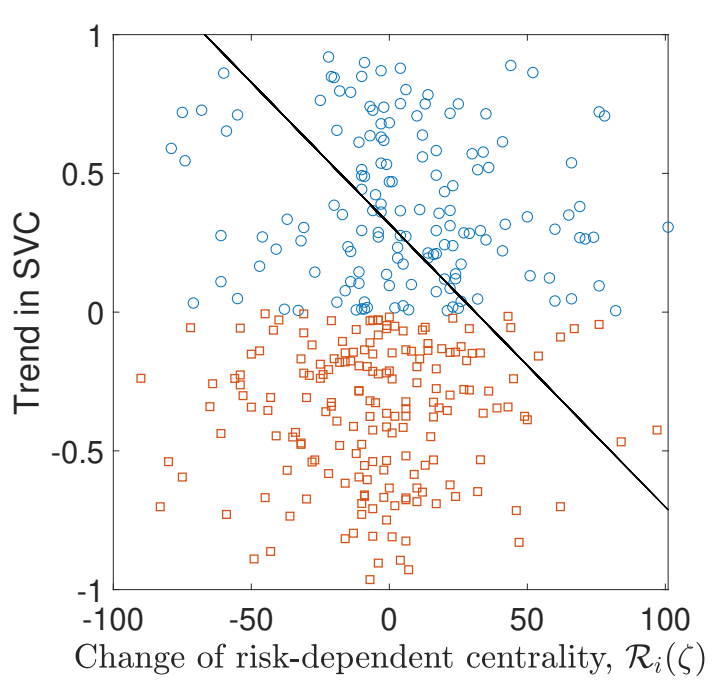

(a)

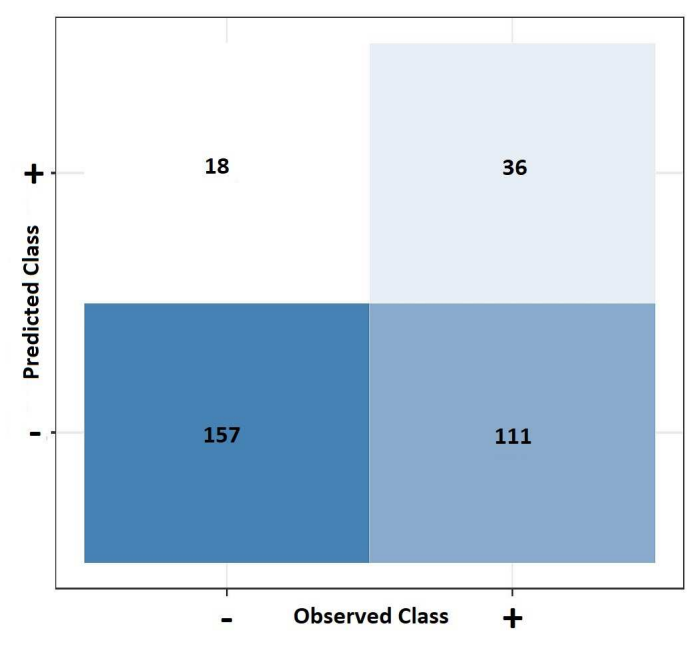

(b)

Figure 12. (a) Illustration of the linear discriminant analysis (LDA) model classifying the trend of corporations into those shrinking their SVC (red squares) and those expanding it (blue circles). The black line represents the LDA model based on the change of $\mathscr{R}_{i}(\zeta)$ for the values of $\zeta=0.01$ and $\zeta=1.0$ to predict the trend in the SVC. Red squares below the line and blue circles over the line are well classified, while blue circles below the line and red squares over the line are wrongly classified. The LDA classifies correctly about 90\% of all companies who shrank their SVC (red squares). (b) Plot of the confusion matrix. On the x-axis we report the true class (Observed Class), and on the y-axis we report the predicted class (Output Class). The number of true negative and true positive cases are on the antidiagonal (bottom and upper parts, respectively), and the number of false negative and false positive cases are on the main diagonal of the matrix (bottom and upper parts, respectively). In the class "minus" ("plus") we consider companies with negative (positive) trend in the SVC for the period 1999-2003.

centrality ranking as $\zeta$ vanishes, it is worth noting that this occurs when the global stress in the market is very low. When the infectability rate is very low, the absolute probability of getting infected also remains very low for both "good" and "bad" companies. To show this fact, let us consider that, according to our model, the probability that a given corporate is not affected by a crisis propagating inside the network is given by $1-x_{i}(t)=\alpha e^{-\frac{\beta}{\alpha}\left(\mathscr{R}_{i}-1\right)}$, where again $\beta$ and $\alpha=1-\beta$ are the initial probabilities to have infected and not-infected nodes, respectively. Hence, the ratio between the probabilities of two nodes $i$ and $j$ to pass successfully through a crisis is given by $e^{\frac{\beta}{\alpha}\left(\mathscr{R}_{j}-\mathscr{R}_{i}\right)}$. We compute these ratios for different couples of corporates operating in a similar sector, a "good" one and a "bad" one (Figure 13).

As expected, at low $\zeta$ the probability of not being infected by a crisis is the same for both high and low risk-centrality companies. But this ratio decreases very quickly as $\zeta$ increases, and this means that for companies that reduced their risk (e.g., Lucent Technologies, Morgan Stanley, Union Carbide, and American Express) the probabilities of staying safe during a crisis are very small if compared with the analogous probabilities for companies that increased their risk (e.g., General Electric, Bank One, Ashland, and Bank of America). 


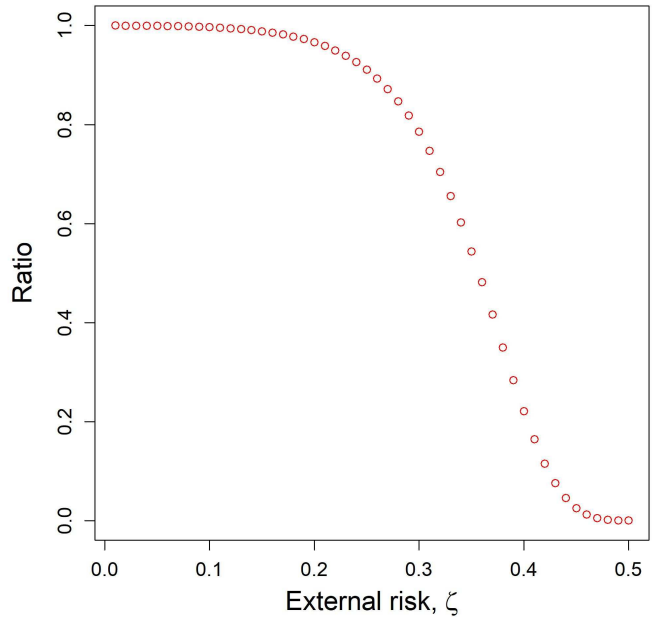

(a)

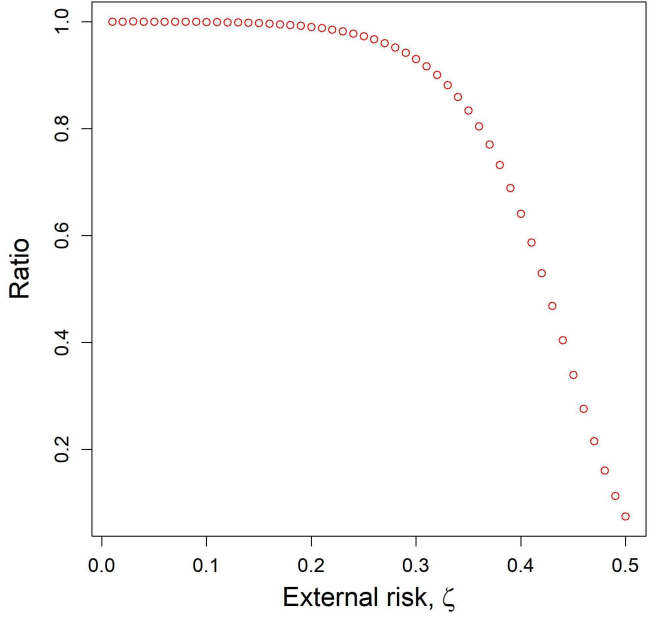

(b)

Figure 13. Figures display the ratios between the probabilities of not being infected by a crisis for two different couples of Corporates: (a) Lucent Technologies Inc. over General Electric Co. (b) Morgan Stanley Co. over Bank One Corp. It is noteworthy that Lucent Technologies Inc. and Morgan Stanley Co. reduced their rankings over time, while General Electric Co. and Bank One Corp. increased their rankings.

7. Ranking interlacement. During the analysis of the two real-world networks studied above, we have noticed that with the change of $\zeta$ some nodes vary their ranking significantly, to the point of changing their positions relative to each other. For instance, in Figure 14 we illustrate six pairs of corporates that interlace their positions with the change of the global infectability in the network. In the first pair, Figure 14(a), we see that at low levels of infectability, i.e., $\zeta \rightarrow 0$, J.P. Morgan\&Co Inc. (red) occupies a position in the ranking of $\mathscr{C}_{i}$ more at the bottom than Bank of America Corp. (blue). That is, at low global infectability J.P. Morgan\&Co is exposed to less risk than Bank of America. However, when the global infectability in the network increases $(\zeta \rightarrow 1)$, Bank of America is exposed to less risk than J.P. Morgan\&Co. A similar interlacement is observed between the other couples in Figure 14. For instance, in Figure 14(f), the interlacement between rankings for General Motors Corp. (red) and Boeing Co. (blue) occurs at a smaller value of $\zeta$ than for the previous cases. Before proceeding with the analysis of this phenomenon, we would like to remark that the existence of ranking interlacement means that the ranking of the nodes in a network based on the risk-dependent centralities is not unique and fixed as in the case of other classical centrality measures, e.g., degree, eigenvector, closeness, betweenness. Here instead the ranking of nodes depends on the global external conditions to which the network is subject.

In order to shed light on the issue of ranking interlacement, we will make use of different representations of the risk-dependent total communicability $\mathscr{R}_{i}(\zeta)$ and circulability $\mathscr{C}_{i}(\zeta)$ measures (the transmissibility is obtained as the difference of these two and can be treated accordingly). First, expanding the matrix exponential in a power series gives the representa- 


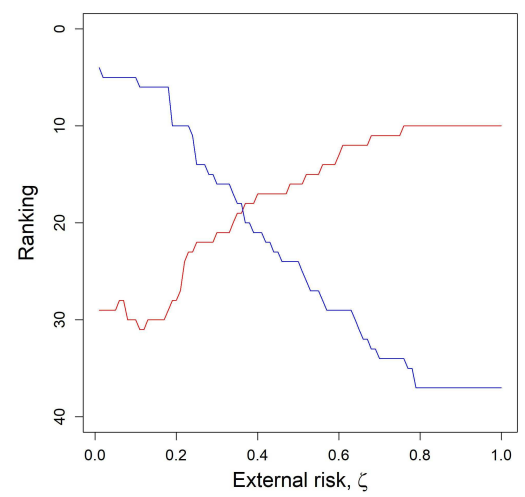

(a)

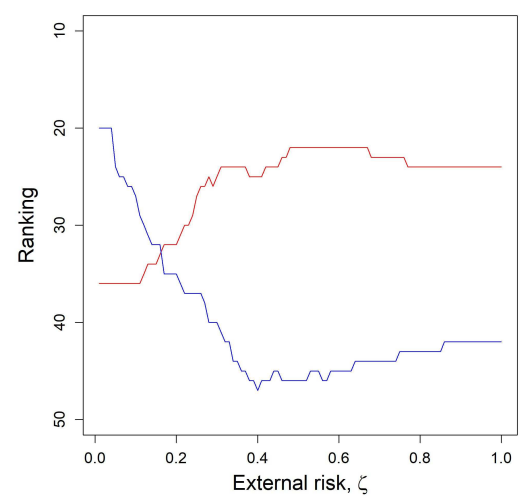

(c)

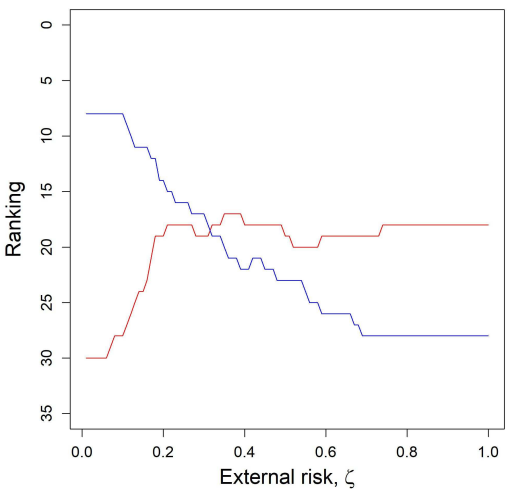

(e)

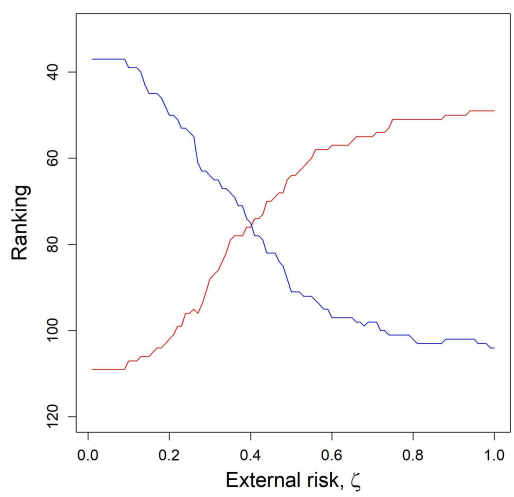

(b)

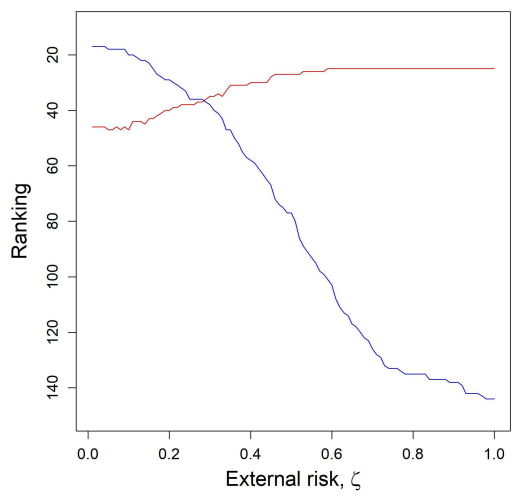

(d)

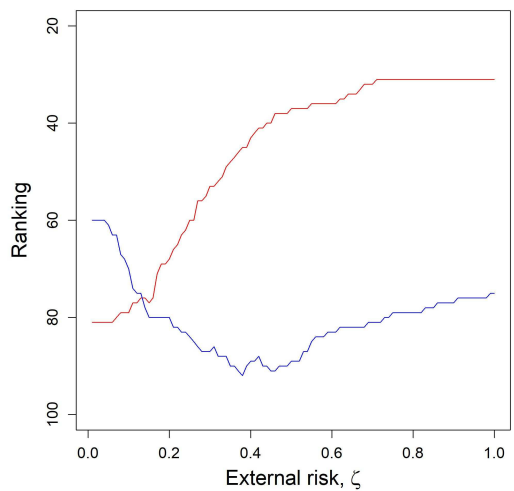

(f)

Figure 14. Illustration of the Circulability Ranking Interlacement for (a) J.P. Morgan\&Co Inc. (red) and Bank of America Corp. (blue), (b) Pfizer Inc. (red) and Ashland Inc. (blue), (c) Morgan Stanley ES Co. (red) and Bank One Corp. (blue), (d) ATEST Corp. (red) and Airtouch Communications Inc. (blue), (e) Union Carbide Corp. New (red) and AON Corp. (blue), (f) General Motors Corp. (red) and Boeing Co. (blue).

Copyright (C) by SIAM. Unauthorized reproduction of this article is prohibited. 
tion

$$
\mathscr{R}_{i}(\zeta)=\left(e^{\zeta A \overrightarrow{1}}\right)_{i}=\sum_{k=0}^{\infty} \frac{\zeta^{k}}{k !} w_{i}^{(k)}
$$

where $w_{i}^{(k)}=\left(A^{k} \overrightarrow{1}\right)_{i}$ denotes the number of walks of length $k$ starting from the node $i$, with $w_{i}^{(0)}=1$. In particular, $w_{i}^{(1)}=k_{i}$, the degree of node $i$. Similarly,

$$
\mathscr{C}_{i}(\zeta)=\left(e^{\zeta A}\right)_{i i}=\sum_{k=0}^{\infty} \frac{\zeta^{k}}{k !} w_{i, i}^{(k)}
$$

where now $w_{i, i}^{(k)}=\left(A^{k}\right)_{i i}$ is the number of closed walks of length $k$ through node $i$; in particular, $w_{i, i}^{(0)}=1, w_{i, i}^{(1)}=0, w_{i, i}^{(2)}=k_{i}$, and $w_{i, i}^{(3)}=2 t_{i}$, where $t_{i}$ is the number of triangles node $i$ participates in.

Second, we recall that the spectral theorem yields the formulas

$$
\mathscr{R}_{i}(\zeta)=\sum_{k=1}^{n} e^{\zeta \lambda_{k}}\left(\psi_{k}^{T} \overrightarrow{1}\right) \psi_{k, i}, \quad \mathcal{C}_{i}(\zeta)=\sum_{k=1}^{n} e^{\zeta \lambda_{k}}\left(\psi_{k, i}\right)^{2} .
$$

Using (7.1)-(7.2), we readily see that both functions of $\zeta$ are absolutely monotonic for $\zeta>0$; i.e., they are positive and infinitely differentiable on $(0, \infty)$, with all the derivatives being nonnegative. In particular, both functions are strictly increasing and strictly convex.

Definition 7.1. We say that the rankings of node $i$ and node $j$ based on the circulability interlace at $\zeta^{*}>0$ if $\mathscr{C}_{i}\left(\zeta^{*}\right)=\mathscr{C}_{j}\left(\zeta^{*}\right)$ and there exists an $\varepsilon>0$ such that $\mathscr{C}_{i}(\zeta)-\mathscr{C}_{j}(\zeta)$ changes sign exactly once in $\left(\zeta^{*}-\varepsilon, \zeta^{*}+\varepsilon\right)$.

In other words, nodes $i$ and $j$ interlace at $\zeta^{*}>0$ if the plots of $\mathscr{C}_{i}(\zeta)$ and $\mathscr{C}_{j}(\zeta)$ cross for $\zeta=\zeta^{*}$. We note that, in principle, it is possible to have $\mathscr{C}_{i}\left(\zeta^{*}\right)=\mathscr{C}_{j}\left(\zeta^{*}\right)$ for some value of $\zeta^{*}$ without interlacing taking place. Two cases are possible: in the first one, the two curves touch at the isolated point $\zeta^{*}$ (without crossing), and in the second one the two functions are identical on an open neighborhood of $\zeta^{*}$ and, therefore, for all $\zeta$ since they are analytic functions. In practice, either scenario is very unlikely to occur, at least for real-world networks. Note that points of tangency must satisfy the additional condition $\mathscr{C}_{i}^{\prime}\left(\zeta^{*}\right)=\mathscr{C}_{j}^{\prime}\left(\zeta^{*}\right)$.

An analogous definition can be given for the ranking based on other $\zeta$-dependent measures, like the total communicability $\mathscr{R}_{i}(\zeta)$. In the following we limit our discussion to the interlacing of rankings according to the circulability, but analogous observations hold for the total communicability and transmissibility functions.

Identifying the interlacing points (if they exist) requires finding the roots of the transcendental equation $\mathscr{C}_{i}(\zeta)-\mathscr{C}_{j}(\zeta)=0$, or

$$
\Psi(\zeta):=\sum_{k=1}^{n} e^{\zeta \lambda_{k}}\left[\psi_{k, i}^{2}-\psi_{k, j}^{2}\right]=0 .
$$

Even if we knew the eigenvalues and eigenvectors of $A$ explicitly, there is no general closed form expression for the roots of the transcendental function $\Psi$. Of course one could 
resort to numerical root-finding techniques, but this would be impractical for large networks. Here and below we give a qualitative discussion followed by a heuristic approach that yields approximations that seem to work well in practice.

We begin with the following result. It applies to both circulability and total communicabilitybased rankings, and in fact to a much larger class of parameter-dependent centrality ranking functions, including Katz centrality [53]. We remind the reader that we restrict the risk rate $\zeta$ to positive values.

Theorem 7.2. Let $i$ and $j$ be two nodes with different eigenvector centrality: $\psi_{1, i} \neq \psi_{1, j}$. Then the number of interlacing points for $i$ and $j$ is necessarily finite (possibly zero).

Proof. Let us assume that there is at least one pair of nodes, $i$ and $j$, whose rankings interlace, so that $\Psi(\zeta)=0$ has at least one positive root. Observe that the ranking of node $i$ provided by $\mathscr{C}_{i}(\zeta)$ is identical to that obtained using

$$
\hat{\mathscr{C}}_{i}(\zeta)=e^{-\zeta \lambda_{1}} \mathscr{C}_{i}(\zeta)=\psi_{1, i}^{2}+\sum_{k=2}^{n} e^{\zeta\left(\lambda_{k}-\lambda_{1}\right)} \psi_{k, i}^{2}
$$

As this quantity tends monotonically to $\psi_{1, i}^{2}$ for $\zeta \rightarrow \infty$, there exists a $\bar{\zeta}$ such that no rank interlacing with node $j$ can occur for $\zeta>\bar{\zeta}$, since all the node rankings must stabilize on the eigenvector rankings in the large $\zeta$ limit. Hence, all interlacing points must fall within the compact interval $[0, \bar{\zeta}]$. Suppose that the number of interlacing points is infinite. By the Bolzano-Weierstrass theorem, this set has a point of accumulation. But since $\hat{\Psi}(\zeta):=$ $e^{-\zeta \lambda_{1}} \Psi(\zeta)$ is analytic, and zero on this set, it must be identically zero everywhere, which contradicts the assumption that there is at least one interlacing point in $(0, \infty)$.

As a consequence we have the following corollary.

Corollary 7.3. If all nodes in the network have different eigenvector centralities, the total number of interlacing points is finite (possibly zero).

A sufficient condition for the existence of at least one interlacing point for the pair of nodes $i$ and $j$ is that $k_{i} \geq k_{j}$ (or $k_{j} \geq k_{i}$ ) while $\psi_{1, i}<\psi_{1, j}$ (resp., $\psi_{1, i}>\psi_{1, j}$ ). This follows from Theorem 4.2: since $\mathscr{C}_{i}(\zeta)$ interpolates smoothly between degree centrality and eigenvector centrality, the only way that a node with higher degree can have lower eigenvector centrality than another node is that the corresponding circulabilities interlace at some value $\zeta^{*}>0$. If more than one interlacing point exists, this number must be odd, for otherwise the node with higher degree would also have higher eigenvector centrality than the other node. That the above condition is not necessary is made clear by considering the possibility of an even number of interlacing points. A necessary condition for the existence of at least one interlacing point is that there exist at least two values of $k$, say $k_{1}$ and $k_{2}$, for which $\left(A^{k_{1}}\right)_{i i}-\left(A^{k_{1}}\right)_{j j}$ and $\left(A^{k_{2}}\right)_{i i}-\left(A^{k_{2}}\right)_{j j}$ have different sign. Indeed, it is obvious from (7.1)-(7.2) that if (say) $\left(A^{k}\right)_{i i} \geq\left(A^{k}\right)_{j j}$ for all $k$, then no rank interlacing point exists. That this condition may not be sufficient is suggested by the fact that the series expansions contain an infinity of terms.

We mention that the same problem has been studied, for a different centrality function (the Katz resolvent), by [56] independently of us.

Copyright (c) by SIAM. Unauthorized reproduction of this article is prohibited. 
7.1. A back-of-the-envelope approach. We now consider heuristics based on truncated series expansions. Let $k_{0} \geq 3$ be the smallest value of $k$ such that the sequence of values $\left\{\left(A^{k}\right)_{i i}-\left(A^{k}\right)_{j j}\right\}_{k \geq 2}$ undergoes a sign change (here zero is considered positive). If no such $k_{0}$ exists, then no interlacing can take place, as we already observed. We consider approximating $\mathscr{C}_{i}(\zeta)$ with its truncation to an order $k \geq k_{0}$ :

$$
\mathscr{C}_{i}(\zeta) \approx 1+\frac{1}{2 !} \zeta^{2} w_{i, i}^{(2)}+\frac{1}{3 !} \zeta^{3} w_{i, i}^{(3)}+\cdots+\frac{1}{k !} \zeta^{k} w_{i, i}^{(k)}=\tilde{\mathscr{C}}_{i}(\zeta)
$$

where we recall that $w_{i, i}^{(k)}=\left(A^{k}\right)_{i i}$. We emphasize that this polynomial approximation assumes that $\zeta$ is small, since the error in it is $O\left(\zeta^{k+1}\right)$. Alternatively we can use, as a surrogate for $\mathscr{C}_{i}$, the same polynomial shifted by 1 and divided by $\zeta^{2}$ :

$$
\frac{\tilde{\mathscr{C}}_{i}(\zeta)-1}{\zeta^{2}}=\frac{1}{2 !} w_{i, i}^{(2)}+\frac{1}{3 !} \zeta w_{i, i}^{(3)}+\cdots+\frac{1}{k !} \zeta^{k-2} w_{i, i}^{(k)},
$$

where now the error is $O\left(\zeta^{k-1}\right)$. We can now use these polynomial approximations to try to locate, approximately, any interlacing points sufficiently small in magnitude. This requires finding the (positive) roots, if any, of the polynomial equation of degree $k-2$ :

$q(\zeta)=\frac{\left(w_{i, i}^{(k)}-w_{j, j}^{(k)}\right)}{k !} \zeta^{k-2}+\frac{\left(w_{i, i}^{(k-1)}-w_{j, j}^{(k-1)}\right)}{(k-1) !} \zeta^{k-3}+\cdots+\frac{\left(w_{i, i}^{(3)}-w_{j, j}^{(3)}\right)}{3 !} \zeta+\frac{\left(w_{i, i}^{(2)}-w_{j, j}^{(2)}\right)}{2 !}=0$.

It is well known that for degree greater than or equal to 5 there is no closed form expression of the solutions of an algebraic equation involving only arithmetic operations and root extractions, so in general if $k \geq 7$, then we will have to resort to numerical methods for solving (7.5). Evaluation of the coefficients requires computing the diagonal entries of powers of the adjacency matrix $A$, which can be expensive for very large graphs and large values of $k$.

As the simplest possible example, we consider the case where $w_{i, i}^{(2)}>w_{j, j}^{(2)}$ and $w_{i, i}^{(3)}<w_{j, j}^{(3)}$ (or vice versa), i.e., $k_{0}=3$. Taking $k=k_{0},(7.5)$ becomes the linear equation

$$
\frac{\left(w_{i, i}^{(3)}-w_{j, j}^{(3)}\right)}{3 !} \zeta+\frac{\left(w_{i, i}^{(2)}-w_{j, j}^{(2)}\right)}{2 !}=0,
$$

which admits the unique solution $\zeta^{*}=\frac{3\left(w_{i, i}^{(2)}-w_{j, j}^{(2)}\right)}{w_{i, i}^{(3)}-w_{j, j}^{(3)}}$, which is of course positive. In terms of the degree of the nodes and the number of triangles in which they take place, this can be written in the form

$$
\zeta^{*}=\frac{3}{2}\left|\frac{k_{i}-k_{j}}{t_{i}-t_{j}}\right|
$$

In the case of weighted networks, the degree is replaced by the weighted degree or strength, and the number of triangles is replaced by the weighted number of cycles of length 3 , i.e., the weight of a cycle of length 3 is the product of the weights at its three edges. A priori, there is no reason to expect that this value is close to an actual interlacing point (assuming it even 
exists), since the behavior of higher order terms may more than offset the influence of the negative term involving $t_{i}-t_{j}$. Better approximations might be obtained by considering higher order approximations; for example, using $k=4$ leads to an easily solved quadratic equation in $\zeta, k=5$ leads to a cubic, and so forth. In any case, these are heuristics whose usefulness can only be assessed experimentally on concrete examples. We emphasize that the use of power series truncation requires knowledge of $k_{0}$, since truncating the series at orders lower than $k_{0}$ would lead to an equation devoid of positive solutions and therefore to concluding that no interlacing points exist for a given pair of nodes, even if such points do exist.

It is also worth recalling Descartes's Rule of Signs, according to which the number of positive real roots of a polynomial (counted with their multiplicities) is equal to the number of sign changes in the (nonzero) coefficients or less than that by an even whole number, when the powers are ordered in descending order. If, moreover, the polynomial is known to have only real roots (as in the case of a symmetric adjacency matrix, i.e., of undirected networks), then the number of sign changes is exactly equal to the number of positive roots. It is then obvious that if the power series is truncated at order $k_{0}$, i.e., as soon as we observe the first sign change in the coefficients, then there will be exactly one positive root, and therefore only one (approximate) interlacing point can be found by this method. A polynomial truncation of higher degree $k>k_{0}$ may have more than one positive root, depending on the number of changes in the coefficients (assuming the network is undirected). We will come back to this case shortly.

To exemplify the previous finding, let us consider a pair of nodes with a small difference in their degree, e.g., $k_{i}-k_{j}=2$; then $-\left(k_{i}-2\right)^{2} \leq\left(t_{i}-t_{j}\right) \leq k_{i}^{2}$, such that if, for instance, $k_{i} \leq 10$ and we let $\zeta$ vary from 0 to 0.1 , we obtain the plot given in Figure 15(a). As can be seen, there are certain values of $\Delta=t_{i}-t_{j}<0$ for which we can obtain positive and negative values of $\mathscr{C}_{i}-\mathscr{C}_{j}$. This is illustrated in Figure $15(\mathrm{~b})$, where we can see that when $-100 \leq \Delta \leq-40$ there are both positive and negative values of $\mathscr{C}_{i}-\mathscr{C}_{j}$. In other words, it is possible to find pairs of nodes for which $\mathscr{C}_{i}\left(\zeta_{1}\right)>\mathscr{C}_{j}\left(\zeta_{1}\right)$ and then $\mathscr{C}_{i}\left(\zeta_{2}\right)<\mathscr{C}_{j}\left(\zeta_{2}\right)$, which means that these nodes will change their ranking position in terms of the risk-dependent centrality when the values of $\zeta$ change even for a relatively narrow window. Notice that if $k_{i}-k_{j}=2$ and $\Delta \geq-30$, such a change is not observed for the corresponding range of $\zeta$ analyzed.

If we now consider a large difference in the node degrees, e.g., $k_{i}-k_{j}=100$, and the same range of change for the difference in the number of triangles, e.g., $-100 \leq \Delta \leq 100$, we do not observe any variation in the ranking of pairs of nodes as can be seen in Figure 16(a). In this case the range of $\Delta$ must be increased dramatically to obtain inversions in the ranking of pairs of nodes (see Figure 16(b)).

To illustrate how well the estimate (7.6) performs, we use it for approximating the interlacement point for several pairs of corporates and compare them with the observed values in Table 2 for the weighted version of the U.S. corporate network.

A few more general considerations on the validity of the power series truncation heuristic can be made. The size of the interval containing any interlacing points is dictated to a large extent by how quickly the rankings based on the measures $\mathscr{C}_{i}(\zeta)$ (or $\tilde{\mathscr{C}}_{i}(\zeta)$ ) stabilize near the rankings obtained using eigenvector centrality. This, in turn, depends on the spectral gap $\lambda_{1}-\lambda_{2}$ : the larger the gap, the faster the eigenvector centrality rankings are approached for 


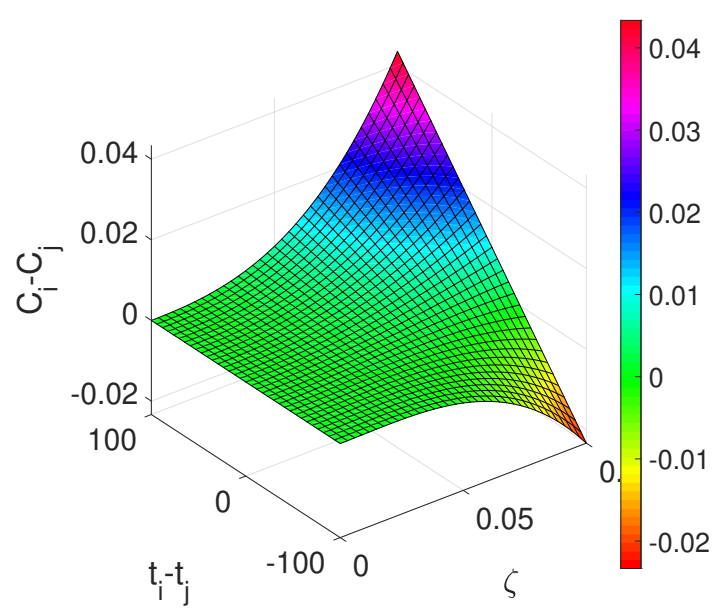

(a)

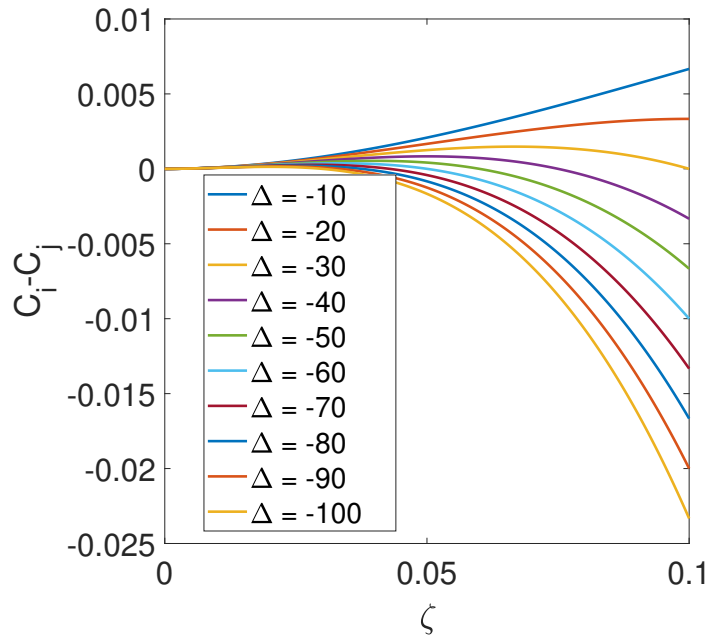

(b)

Figure 15. (a) Illustration of the change in the difference in the risk-dependent centrality of nodes having a small difference in degrees, $k_{i}-k_{j}=2$, as a function of the difference in the number of triangles, $t_{i}-t_{j}$, and of the network infectivity risk $\zeta$. (b) Some of the curves obtained for $k_{i}-k_{j}=2$ and a given value of $\Delta=t_{i}-t_{j}$ as a function of $\zeta$.

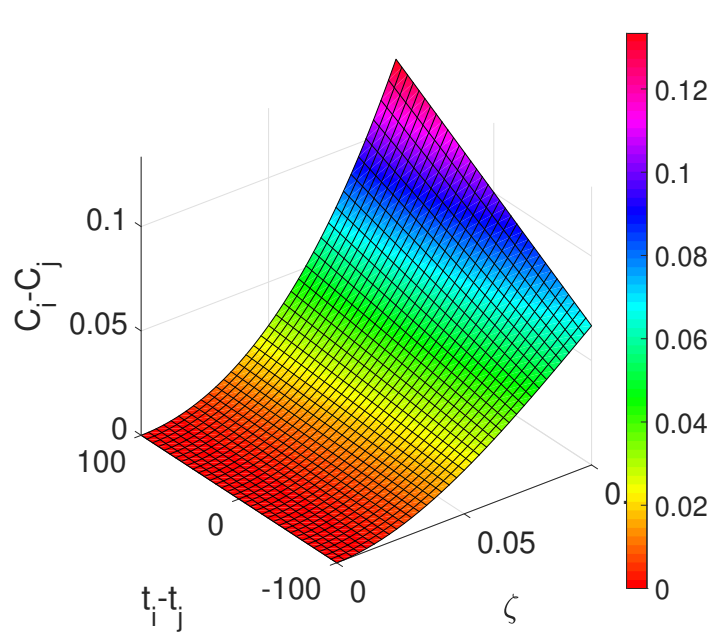

(a)

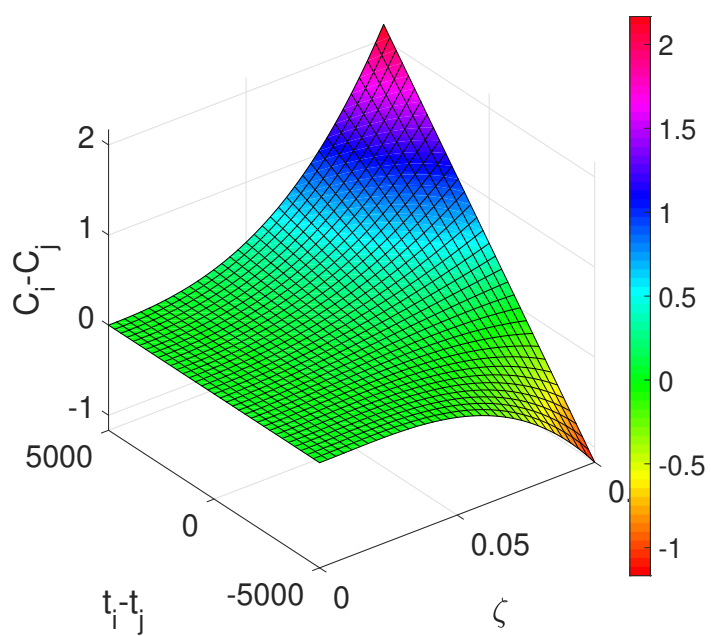

(b)

Figure 16. Illustration of the change in the difference in the risk-dependent centrality of nodes having a small difference in degrees, $k_{i}-k_{j}=100$, as a function of the difference in the number of triangles, $-100 \leq$ $\Delta \leq 100$ (a) and $-5000 \leq \Delta \leq 5000$ (b), and of the network infectivity (risk) $\zeta$.

increasing values of $\zeta$. Hence, in the case of relatively large gaps, we expect any interlacing values to occur for fairly small values of $\zeta$. In this case, the heuristics based on polynomial approximations may be justified, since interlacing is likely to occur already for small values of $\zeta$. As is well known, however, it is not easy to determine when the spectral gap is "sufficiently

Copyright (C) by SIAM. Unauthorized reproduction of this article is prohibited. 
Table 2

Calculation of the crossing point of ranking interlacement for several pairs of corporates in the U.S. corporates network of 1999 as well as the observed values at which such interlacements occur.

\begin{tabular}{|c|l|l|c|c|}
\hline Plot & Corporate 1 & Corporate 2 & $\zeta^{*}$ calculated & $\zeta^{*}$ observed \\
\hline \hline (a) & J.P. Morgan\&Co Inc. & Bank of America Corp. & 0.375 & 0.37 \\
\hline (b) & Pfizer Inc. & Ashland Inc. & 0.441 & 0.41 \\
\hline (c) & Morgan Stanley \& Co. & Bank One Corp. & 0.176 & 0.17 \\
\hline (d) & AT\&T Corp. & Airtouch Communications & 0.273 & 0.27 \\
\hline (e) & Union Carbide Corp. New & AON Corp. & 0.353 & 0.32 \\
\hline (f) & General Motors Corp. & Boeing Co. & 0.214 & 0.14 \\
\hline
\end{tabular}

large." On the other hand, when the spectral gap is tiny, the interval $[0, \bar{\zeta}]$ is going to be larger, and therefore there is "more room" for the occurrence of interlacing. Unfortunately, in this case it is not clear that polynomial truncation will be effective in approximately locating the interlacing points. In this case, a possible solution is to expand the functions $\mathscr{C}_{i}(\zeta)$ not only around the value $\zeta=0$ but also around a few values $\zeta_{0}>0$. This strategy can also be used to find a possible second point of interlacing after having found a first such point $\zeta^{*}$. Expanding around $\zeta^{*}$ leads to

$$
\begin{aligned}
& \Psi\left(\zeta^{*}+\eta\right)=\mathscr{C}_{i}\left(\zeta^{*}+\eta\right)-\mathscr{C}_{j}\left(\zeta^{*}+\eta\right) \\
& =\frac{1}{2 !}\left(w_{i, i}^{(2)}-w_{j, j}^{(2)}\right) \eta^{2}+\frac{1}{3 !}\left(w_{i, i}^{(3)}-w_{j, j}^{(3)}\right) \eta^{3}+\cdots+\frac{1}{k !}\left(w_{i, i}^{(k)}-w_{j, j}^{(k)}\right) \eta^{k}+O\left(\eta^{k+1}\right) .
\end{aligned}
$$

Dividing by $\eta^{2}$ and setting the result equal to zero leads to an algebraic equation of degree $k-2$ for $\eta$; the smallest positive root $\eta^{*}$ of this equation, if there are any, leads to the approximation $\zeta^{*}+\eta^{*}$ for the next interlacing point, and so forth.

Completely analogous considerations apply to the approximation of interlacing points when the ranking of nodes is done according to the risk-based total communicability measure $\mathscr{R}_{i}(\zeta)$. In this case the transcendental equation to be solved is given by

$$
\chi(\zeta)=\mathscr{R}_{i}(\zeta)-\mathscr{R}_{j}(\zeta)=\sum_{k=1}^{n} e^{\zeta \lambda_{k}}\left(\psi_{k}^{T} \overrightarrow{1}\right)\left[\psi_{k, i}-\psi_{k, j}\right]=0 .
$$

Let $w_{i}^{(k)}=\left(A^{k} \overrightarrow{1}\right)_{i}$. Then, truncating the series expansion (7.1) and dividing by $\zeta>0$ leads to the approximation

$$
\frac{\left(w_{i}^{(k)}-w_{j}^{(k)}\right)}{k !} \zeta^{k-1}+\cdots+\frac{\left(w_{i}^{(2)}-w_{j}^{(2)}\right)}{2 !} \zeta+\left(w_{i, i}^{(2)}-w_{j, j}^{(2)}\right)=0
$$

for the equation whose smallest positive solution approximates the first interlacement value for the rankings of nodes $i$ and $j$, assuming it exists; here again $k \geq k_{0}$, where now $k_{0} \geq 2$ is the smallest integer value for which the sequence $\left\{w_{i}^{(k)}-w_{i}^{(k)}\right\}_{k}$ changes sign. The simplest possible case is when $k=k_{0}=2$, which occurs when $w_{i, i}^{(2)}-w_{j, j}^{(2)}$ and $w_{i}^{(2)}-w_{j}^{(2)}=\left(A^{2} \overrightarrow{1}\right)_{i}-\left(A^{2} \overrightarrow{1}\right)_{j}$ have different sign. In this case (7.7) reduces to the linear equation

$$
\frac{\left(w_{i}^{(2)}-w_{j}^{(2)}\right)}{2} \zeta+\left(w_{i, i}^{(2)}-w_{j, j}^{(2)}\right)=0,
$$

Copyright (c) by SIAM. Unauthorized reproduction of this article is prohibited. 
with the unique root

$$
\zeta^{*}=2 \frac{w_{i, i}^{(2)}-w_{j, j}^{(2)}}{w_{j}^{(2)}-w_{i}^{(2)}}>0 .
$$

8. Risk prediction and COVID-19. Since we submitted the first versions of this work, a pandemic has been expanding from the city of Wuhan, in the Hubei province of China [83, 84], starting in December 2019. This disease is produced by a new coronavirus named SARS-CoV-2 [45] and has affected in about three months more than 200 countries around the world. The main problem right now is of a medical nature, but as stated by Baldwin and Weder di Mauro this coronavirus is "as contagious economically as it is medically" [4]. One of the most important characteristics of this pandemic in comparison with recent ones is that it is hitting very strongly the most important economies in the world: China, USA, Germany, Italy, and Spain. There are some preliminary studies about the macroeconomic impacts of this pandemic (see, for instance, [4]). However, it is important to apply mathematical and computational techniques to forecast, at regional, national, and international levels, the impact of this crisis on financial institutions, corporations, and small companies. All of them are highly interconnected in a globally dependent economy, forming series of complex networks. In this new scenario the current work represents an opportunity for modelers to advance predictions on the potential risks different institutions are subject to in the current situation. This modeling scenario consists of the networks of interactions between the institutions under analysis assuming a high infectability in the network. Using the transmissibility and circulability measures defined here, the modeler can understand how at risk of transmitting the crisis to others or, respectively, of staying in a cycle of repeated economic difficulties, a company is. At the same time, the current work allows modeling of how different palliative measures taken by regional or global financial institutions in the European Union, USA, or China can impact these companies. In this case, the modeler should drop the infectivity of the system and analyze how the ranking of risk for the different companies changes to gain insights about their potential recovery or bankruptcy.

9. Conclusions. In general, node centrality in networks is of either of two types: (i) node centrality in networks of time-invariant topology [29], or (ii) node centrality in networks of time-dependent topology (a.k.a. temporal networks) [49]. In this work we have developed a new concept of node centrality, depending on both the topology of the network and the external conditions to which the network as a whole is subjected. In particular, we have focused on global risk as the external factor by which an economic and financial network is affected. We started by considering the "Susceptible-Infected" model and its connection to the communicability functions of nodes and edges in a network. Then, we developed a few centrality measures which depend not only on the local and global topological environment of a node but also on the level of infectivity stressing the system as a whole. In this way we have been able to make predictions in financial and economic systems about the changes in the risk-dependent centralities of nodes as a function of the global level of infectivity in the system. We observe that without altering the topology of the network, i.e., without varying any connection between the nodes, the ranking of the nodes, according to these new centrality measures, changes significantly as the infectivity rate changes. In the real-world networks 
studied here we have been able to associate those changes in the risk-dependent centrality of nodes with events of the real financial and economic worlds in which these networks are embedded. In closing, we provide here theoretical, computational, and empirical evidence that the node centrality is not a static function even when the topology of the system is not varying at all. This new paradigm is expected to play a fundamental role in assessing the robustness of financial and economic systems to the variation of the external conditions which they are subjected to.

Appendix A. Risk-dependent centrality measures for complete graphs. The following theorem provides a close expression for $\mathscr{R}_{i}, \mathscr{C}_{i}$, and $\mathscr{T}_{i}$ for a complete network.

Theorem A.1. The risk-dependency $\mathscr{R}_{i}$ for each node in a complete graph is given by

$$
\mathscr{R}_{i}=e^{(n-1) \zeta},
$$

and the circulability and transmissibility are given by

$$
\mathscr{C}_{i}(\zeta)=\frac{n-1}{n}\left[\frac{e^{(n-1) \zeta}}{n-1}+\frac{1}{e^{\zeta}}\right], \quad \mathscr{T}_{i}(\zeta)=\frac{n-1}{n}\left[e^{(n-1) \zeta}-\frac{1}{e^{\zeta}}\right] .
$$

Proof. For a complete graph, $\psi_{j}^{T} \cdot \overrightarrow{1}=0, j \neq 1$, because of the mutual orthogonality between $\psi_{j}, j \neq 1$, and the principal eigenvector $\psi_{1}$ of constant components. That is, $\mathscr{R}_{i}$ is completely determined by the eigenvector centralities $\psi_{1, i}$, which of course are equal for every node and equal to $\psi_{1, i}=\frac{1}{\sqrt{n}}$. Since $\lambda_{1}=n-1$, we obtain

$$
\mathscr{R}_{i}=e^{\zeta \lambda_{1}}\left(\psi_{1}^{T} \cdot \overrightarrow{1}\right) \psi_{1, i}+0=e^{(n-1) \zeta}\left(\frac{1}{\sqrt{n}} \cdot n\right) \frac{1}{\sqrt{n}}=e^{(n-1) \zeta} .
$$

Subgraph centrality close expression for a complete graph is provided in [32]:

$$
\mathscr{C}_{i}(1)=S C(i)=\frac{1}{n}\left[e^{n-1}+\frac{n-1}{e}\right] .
$$

Multiplying each entry in $A$ by $\zeta$ and summing up the power series, we get

$$
\mathscr{C}_{i}(\zeta)=\frac{n-1}{n}\left[\frac{e^{(n-1) \zeta}}{n-1}+\frac{1}{e^{\zeta}}\right] .
$$

By difference, we get $\mathscr{T}_{i}(\zeta)$.

An important remark concerns the ratio $\frac{\mathscr{C}_{i}}{\mathscr{R}_{i}}$. Indeed,

$$
\lim _{\zeta \rightarrow+\infty} \frac{\mathscr{C}_{i}}{\mathscr{R}_{i}}=\lim _{\zeta \rightarrow+\infty} \frac{\frac{n-1}{n}\left[\frac{e^{(n-1) \zeta}}{n-1}+\frac{1}{e^{\zeta}}\right]}{e^{(n-1) \zeta}}=\lim _{\zeta \rightarrow+\infty}\left[\frac{1}{n}+\frac{n-1}{n} \frac{1}{e^{n \zeta}}\right]=\frac{1}{n} .
$$

Similarly,

$$
\lim _{\zeta \rightarrow+\infty} \frac{\mathscr{C}_{i}}{\mathscr{T}_{i}}=\frac{\frac{e^{(n-1) \zeta}}{n-1}+\frac{1}{e^{\zeta}}}{e^{(n-1) \zeta}-\frac{1}{e^{\zeta}}}=\frac{1}{n-1}
$$

Copyright $\odot$ by SIAM. Unauthorized reproduction of this article is prohibited. 


\section{REFERENCES}

[1] F. Allen And A. Babus, Networks in finance, in The Network Challenge: Strategy, Profit and Risk in an Interlinked World, Wharton School Publishing, Upper Saddle River, NJ, 2009.

[2] R. Alquist, R. Mukherjee, And L. Tesar, Fire-sale FDI or Business as Usual?, Working Paper, National Bureau of Economic Research, 2013.

[3] H. Amini, R. Cont, And A. Minca, Resilience to contagion in financial networks, Math. Finance, 26 (2016), pp. 329-365.

[4] R. Baldwin and B. Weder di Mauro, eds., Economics in the Time of COVID-19, CEPR Press, London, 2020.

[5] A. Barja, A. Martínez, A. Arenas, P. Fleurquin, J. Nin, J. J. Ramasco, and E. Tomás, Assessing the risk of default propagation in interconnected sectoral financial networks, EPJ Data Sci., 8 (2019), 32 .

[6] S. Battiston, D. Delli Gatti, M. Gallegati, B. Greenwald, and J. E. Stiglitz, Liaisons dangereuses: Increasing connectivity, risk sharing, and systemic risk, J. Econ. Dyn. Control, 36 (2012), pp. 1121-1141.

[7] S. Battiston, D. Delli Gatti, M. Gallegati, B. Greenwald, and J. E. Stiglitz, Default cascades: When does risk diversification increase stability?, J. Financ. Stabil., 8 (2012), pp. 138-149.

[8] S. Battiston, M. Puliga, R. Kaushik, P. Tasca, and G. Caldarelli, DebtRank: Too central to fail? Financial networks, the FED and systemic risk, Sci. Rep. 2, 541 (2012), pp. 1-6.

[9] M. Benzi And P. Boito, Quadrature rule-based bounds for functions of adjacency matrices, Linear Algebra Appl., 433 (2010), pp. 637-652.

[10] M. Benzi And C. F. Klymko, Total communicability as a centrality measure, J. Complex Netw., 1 (2013), pp. 124-149.

[11] M. BenzI AND C. F. Klymko, On the limiting behavior of parameter-dependent network centrality measures, SIAM J. Matrix Anal. Appl., 36 (2015), pp. 686-706, https://doi.org/10.1137/130950550.

[12] P. Bongini, G. P. Clemente, And R. Grassi, Interconnectedness, G-SIBs and network dynamics of global banking, Finance Res. Lett., 27 (2018), pp. 185-192.

[13] M. Boss, H. Elsinger, M. Summer, And S. Thurner, An Empirical Analysis of the Network Structure of the Austrian Interbank Market, Financial Stability Report, Oesterreichische National Bank, 2004.

[14] A. Bucci, D. La Torre, D. Liuzzi, and S. Marsiglio, Financial contagion and economic development: An epidemiological approach, J. Econ. Behavior Org., 162 (2019), pp. 211-28.

[15] J. Cocco, F. Gomes, And N. Martins, Lending relationships in the interbank market, J. Financ. Intermed., 18 (2009), pp. 24-48.

[16] L. Cohen, A. Frazzini, AND C. MAlloy, The small world of investing: Board connections and mutual fund returns, J. Political Econ., 116 (2008), pp. 951-979.

[17] R. Cont, A. Moussa, And E. B. Santos, Network structure and systemic risk in banking systems, in Handbook of Systemic Risk, J. P. Fouque and J. Langsam, eds., Cambridge University Press, Cambridge, UK, 2013, pp. 327-368.

[18] M. Corominas-Bosch, Bargaining in a network of buyers and sellers, J. Econ. Theory, 115 (2004), pp. $35-77$.

[19] T. M. Cover and J. A. Thomas, Elements of Information Theory, Wiley \& Sons, New York, 2006

[20] G. F. Davis, M. Yoo, And W. E. BAKer, The small world of the American corporate elite, 1982-2001, Strategic Organization, 1 (2003), pp. 301-26.

[21] N. Demiris, T. Kypraios, and L. V. Smith, On the epidemic of financial crises, J. Roy. Statist. Soc. Ser. A, 177 (2014), pp. 697-723.

[22] K. Dimitrios And O. Vasileios, A network analysis of the Greek stock market, Procedia Econ. Financ., 33 (2015), pp. 340-9.

[23] N. Economides, The economics of networks, Int. J. Ind. Organ., 14 (1996), pp. 673-99.

[24] S. Edwards, Capital Flows and the Emerging Economies: Theory, Evidence, and Controversies, National Bureau of Economic Research Conference Report Series, University of Chicago Press, Chicago, IL, 2008.

[25] H. Elsinger, A. LeHAR, AND M. Summer, Using market information for banking systems, Int. J. Central Bank., 27 (2006), pp. 137-165.

Copyright (C) by SIAM. Unauthorized reproduction of this article is prohibited. 
[26] L. Erdős, A. Knowles, H.-T. YaU, And J. Yin, Spectral statistics of Erdős-Rényi graphs I: Local semicircle law, Ann. Probab., 41 (2013), pp. 2279-2375.

[27] P. ERdős And A. RÉnyi, On the evolution of random graphs, Publ. Math. Inst. Hungar. Acad. Sci., 5 (1960), pp. 17-61.

[28] P. ERdős AND A. RÉNYI, On the strength of connectedness of a random graph, Acta Math. Acad. Sci. Hungar., 12 (1961), pp. 261-267.

[29] E. Estrada, The Structure of Complex Networks: Theory and Applications, Oxford University Press, Oxford, UK, 2012.

[30] E. Estrada And N. Hatano, Communicability in complex networks, Phys. Rev. E., 77 (2008), 036111.

[31] E. Estrada And N. Hatano, A vibrational approach to node centrality and vulnerability in complex networks, Phys. A Stat. Mech. Appl., 389 (2010), pp. 3648-3660.

[32] E. Estrada and J. A. Rodríguez-Velázquez, Subgraph centrality in complex networks, Phys. Rev. E (3), 71 (2005), 056103.

[33] M. Fafchamps And F. Gubert, The formation of risk sharing networks, J. Dev. Econ., 83 (2007), pp. 326-350.

[34] M. Fafchamps And S. Lund, Risk-sharing networks in rural Philippines, J. Dev. Econ., 71 (2003), pp. 261-287.

[35] P. FeRnANDEz ANd L. Reinoso, Shareholder value creators in the S\&SP 500: Year 2003, SSRN Electron. J., https://doi.org/10.2139/ssrn.506102, 2004.

[36] E. FisheR, A biological approach for financial network contagion based on the Susceptible-InfectedRecovered (SIR) model, Análisis Económico, 28 (2013), pp. 109-128.

[37] T. Furusawa and H. Konishi, Free trade networks, J. Int. Econ., 72 (2007), pp. 310-35.

[38] P. Gai, A. G. Haldane, And S. Kapadia, Complexity, concentration and contagion, J. Monetary Econ., 58 (2011), pp. 453-470.

[39] P. Gai and S. Kapadia, Contagion in financial networks, Proc. R. Soc. Lond. Ser. A Math. Phys. Eng. Sci., 466 (2010), pp. 2401-2423.

[40] D. M. Gale And S. Kariv, Financial networks, Am. Econ. Rev., 97 (2007), pp. 99-103.

[41] A. Galeotti and S. Goyal, A Theory of Strategic Diffusion, Working Paper, 2007.

[42] A. Garas, P. Argyrakis, C. Rozenblat, M. Tomassini, and S. Havlin, Worldwide spreading of economic crisis, New J. Phys., 12 (2010), 113043.

[43] P. Glasserman and H. P. Young, How likely is contagion in financial networks?, J. Bank Finance, 50 (2015), pp. 383-399.

[44] R. Glick And A. Rose, Contagion and trade: Why are currency crises regional?, J. Int. Money Finance, 18 (1999), pp. 603-617.

[45] A. Gorbalenya, S. Baker, R. Baric, Et Al., Coronaviridae study group of the International Committee on Taxonomy of Viruses: The species Severe acute respiratory syndrome-related coronavirus: Classifying 2019-nCoV and naming it SARS-CoV-2, Nat. Microbiol., 5 (2020), pp. 536-544.

[46] B. Golub And M. O. Jackson, Nave learning in social networks: Convergence, influence, and the wisdom of crowds, Amer. Econom. J. Microeconom., 2 (2010), pp. 112-149.

[47] S. Goyal and J. L. Moraga-Gonzalez, RESD networks, Rand J. Econ., 1 (2001), pp. 686-707.

[48] A. G. Haldane And R. M. MaY, Systemic risk in banking ecosystems, Nature, 469 (2011), pp. 351-355.

[49] P. Holme and J. Saramaki, Temporal networks, Phys. Rep., 519 (2012), pp. 97-125.

[50] I. Hull, The development and spread of financial innovations, Quant. Econ., 7 (2016), pp. 613-636.

[51] S. Janson, T. Luczak, And A. Rucinski, Random Graphs, Wiley, New York, 2000.

[52] M. KAnno, Assessing systemic risk using interbank exposures in the global banking system, J. Financ. Stabil., 20C (2015), pp. 105-130.

[53] L. KATz, A new status index derived from sociometric data analysis, Psychometrika, 18 (1953), pp. 39-43.

[54] M. L. Katz And C. Shapiro, Systems competition and network effects, J. Econ. Perspect., 8 (1994), pp. 93-115.

[55] A. KiRman, The economy as an evolving network, J. Evol. Econ., 7 (1997), pp. 339-53.

[56] K. Kloster, Talk delivered at the International Conference on Industrial and Applied Mathematics (ICIAM 2019), Valencia, Spain, 2019, and personal communication.

[57] A. Knowles and R. Rosenthal, Eigenvalue confinement and spectral gap for random simplicial complexes, Random Struct. Alg., 51 (2017), pp. 506-537.

Copyright (C) by SIAM. Unauthorized reproduction of this article is prohibited. 
[58] O. Kostylenko, H. S. Rodrigues, And D. F. M. Torres, The spread of a financial virus through Europe and beyond, AIMS Math., 4 (2019), pp. 86-98.

[59] F. Kramarz and D. Thesmar, Social networks in the boardroom, J. Eur. Econ. Assoc., 11 (2013), pp. 780-807.

[60] R. E. Kranton and D. F. Minehart, A theory of buyer-seller networks, Am. Econ. Rev., 91 (2001), pp. $485-508$.

[61] W. Lazonick and M. O'Sullivan, Maximizing shareholder value: A new ideology for corporate governance, Econ. Soc., 29 (2010), pp. 13-35.

[62] C. H. Lee, S. Tenneti, And D. Y. Eun, Transient Dynamics of Epidemic Spreading and Its Mitigation on Large Networks, preprint, https://arxiv.org/abs/1903.00167, 2019.

[63] K. M. Lee, J. S. Yang, G. Kim, J. Lee, K. I. Goh, And I. M. Kim, Impact of the topology of global macroeconomic network on the spreading of economic crises, PLoS One, 6 (2011), e18443.

[64] D. López-Pintado, Diffusion in complex social networks, Games Econ. Behav., 62 (2008), pp. 573-590.

[65] R. N. Mantegna, Hierarchical structure in financial markets, Eur. Phys. J. B, 11 (1999), pp. $193-197$.

[66] R. M. May, S. A. Levin, And G. Sugihara, Complex systems: Ecology for bankers, Nature, 451 (2008), pp. 893-895.

[67] W. Mei, S. Mohagheghi, S. Zampieri, And F. Bullo, On the dynamics of deterministic epidemic propagation over networks, Annu. Rev. Control, 44 (2017), pp. 116-128.

[68] D. Mugnolo, Dynamical systems associated with adjacency matrices, Discrete Contin. Dyn. Syst. Ser. B, 23 (2018), pp. 1945-1973.

[69] M. E. Newman, A measure of betweenness centrality based on random walks, Soc. Netw., 27 (2005), pp. $39-54$.

[70] M. Nekovee, Y. Moreno, G. Bianconi, and M. Marsili, Theory of rumour spreading in complex social networks, Phys. A Stat. Mech. Appl., 374 (2007), pp. 457-470.

[71] B. Nguyen-Dang, Does the Rolodex Matter? Corporate Elite's Small World and the Effectiveness of Boards of Directors, SSRN Working Paper, 2007.

[72] J. P. Onnela, A. Chakraborti, K. Kaski, J. Kertész, and A. Kanto, Dynamics of market correlations: Taxonomy and portfolio analysis, Phys. Rev. E, 68 (2003), 056110.

[73] R. Pastor-Satorras, C. Castellano, P. Van Mieghem, and A. Vespignani, Epidemic processes in complex networks, Rev. Modern Phys., 87 (2015), pp. 925-979.

[74] R. Peckham, Contagion: Epidemiological models and financial crises, J. Public Health, 36 (2014), pp. $13-17$.

[75] G. Peralta And A. Zareei, A network approach to portfolio selection, J. Empir. Finance, 38 (2016), pp. 157-180.

[76] D. Philippas, Y. Koutelidakis, and A. Leontitsis, Insights into European interbank network contagion, Managerial Finance, 41 (2015), pp. 754-772.

[77] F. Pozzi, T. Di Matteo, And T. Aste, Spread of risk across financial markets: Better to invest in the peripheries, Sci. Rep., 3 (2013), 1665.

[78] C. Puhr, R. Seliger, And M. Sigmund, Contagiousness and Vulnerability in the Austrian Interbank Market, Financial Stability Report 24, Oesterreichische National Bank, 2012.

[79] M. Tirado, Complex network for a crisis contagion on an international system, Int. J. Modern Phys. C, 23 (2012), 1250058.

[80] M. Tolvanen, Contagion in the Interbank Network: An Epidemiological Approach, Bank of Finland Research Discussion Paper, 2013.

[81] P. Van Mieghem, K. Devriendt, and H. Cetinay, Pseudoinverse of the Laplacian and best spreader node in a network, Phys. Rev. E., 96 (2017), 032311.

[82] V. H. VU, Spectral norm of random matrices, in Proceedings of the 37th Annual ACM Symposium on Theory of Computing, STOC '05, ACM, New York, 2005, pp. 423-430.

[83] F. Wu, S. Zhao, B. Yu, Y. M. Chen, W. Wang, Z. G. Song, Y. Hu, Z. W. Tao, J. H. Tian, Y. Y. PeI, AND M. L. YUAN, A new coronavirus associated with human respiratory disease in China, Nature, 579 (2020), pp. 265-269.

[84] P. Zhou, X. L. Yang, X. G. Wang, B. Hu, L. Zhang, W. Zhang, H. R. Si, Y. Zhu, B. Li, C. L. HuAng, AND H. D. Chen, A pneumonia outbreak associated with a new coronavirus of probable bat origin, Nature, 579 (2020), pp. 270-273.

Copyright ( by SIAM. Unauthorized reproduction of this article is prohibited. 